\title{
GAIT PATTERN CLASSIFICATION OF CHILDREN WITH DEVELOPMENTAL COORDINATION DISORDER
}

\author{
by
}

Sarah Jean Woodruff

B.P.E., University of New Brunswick, 1998

A thesis submitted in partial fulfilment of the requirements for the Degree of

M.Sc. (Exercise \& Sport Science)

in the Graduate Academic Unit of Kinesiology
Supervisor(s): $\quad$ Connic Bothwell-Myers, Ph.D., Kinesiology
Maureen Tingley, Ph.D., Mathematics and Statistics
Therese Brisson, Ph.D., Kinesiology

Examining Board: Connie Bothwell-Myers, Ph.D., Kinesiology

Wayne Albert, Ph.D., Kinesiology

Edmund Biden, D.Phil., Mechanical Engineering

\section{THE UNIVERSITY OF NEW BRUNSWICK}

\author{
August, 2000
}

() Sarah Woodruff, 2000 
National Library

of Canada

Acquisitions and Bibliographic Services

395 Wellington Stroet Onawa ON K1A ONA Canada
Bibliothèque nationale du Canada

Acquisitions et services bibliographiques

395. ne Wellington

Onawa ON KIA ONA

Canada
The author has granted a nonexclusive licence allowing the National Library of Canada to reproduce, loan, distribute or sell copies of this thesis in microform, paper or electronic formats.

The author retains ownership of the copyright in this thesis. Neither the thesis nor substantial extracts from it may be printed or otherwise reproduced without the author's permission.
L'auteur a accordé une licence non exclusive permettant à la Bibliothèque nationale du Canada de reproduire, prêter, distribuer ou vendre des copies de cette thèse sous la forme de microfiche/film, de reproduction sur papier ou sur format électronique.

L'auteur conserve la propriété du droit d'auteur qui protège cette thèse. $\mathrm{Ni}$ la thèse ni des extraits substantiels de celle-ci ne doivent être imprimés ou autrement reproduits sans son autorisation. 


\begin{abstract}
A healthy lifestyle, including regular physical activity, is being promoted in Canada as it has been shown to reduce the risk of cardiovascular disease, osteoporosis, stroke and depression (Health Canada, 1999). Creating positive movement experiences throughout childhood will most likely promote an active lifestyle into adulthood. On the other hand, negative movement experiences will hinder a child's experience and that individual will likely refrain from taking part in sports and games altogether. Researchers have therefore become interested in children with Developmental Coordination Disorder (DCD), as children with DCD are unable to execute movement patterns with complete confidence.
\end{abstract}

The purpose of this study was to validate Wilson's (1998) statistical procedure of classifying the gait patterns of a population, DCD, that may not be so abnormal using naked eye observation. A secondary objective of this research was to formulate another one-dimensional measure of normal gait (Fscore) using the time/distance variables, as more often these variables are easier and less expensive to measure. The statistical procedures were formulated using normal children from the San Diego Children's Hospital study by Sutherland, Olshen, Biden, and Wyatt (1988). Revie and Larkin (1993) indicated "children who look awkward and are described as clumsy, dyspraxic, or poorly coordinated have difficulty acquiring and performing basic movement patterns such as walking, running, hopping, jumping, throwing, 
catching, kicking, and hitting" (p. 29). Therefore, children with DCD are in a perfect position to determine the power of Wilson's (1998) statistical procedure.

A total of 333 children, from 5 schools, were considered for the DCD group. Homeroom teachers nominated and filled out the MABC Checklist on those students who they thought may have problems with fine and/or gross motor abilities. The MABC Test was administered to 16 subjects for whom the teacher had nominated and parental consent was obtained. A total of 11 subjects were diagnosed with DCD using the MABC Test and invited to the Gait Laboratory at UNB.

The Fscore was formulated using gait data on 178 normal children (aged 3 - 7 years old) included in the San Diego database (Sutherland et al., 1988). The Fscore used four measures: percentage of opposite toe off, percentage of single stance, percentage of toe off, and step length as a percentage of stride length.

The gait patterns of seven subjects with DCD (six male and one female) were analyzed using the Wilson score and the Fscore. The Wilson score classified two of the seven DCD trials as abnormal. The Fscore was able to classify all but one DCD trial as abnormal. The new Fscore was more sensitive to detect subtle abnormalities as more children with DCD were classified as abnormal. This type of gait classification is significant in guiding identification and intervention strategies. 


\section{ACKNOWLEDGEMENT}

I would like to take the opportunity to thank certain individuals who have made this thesis possible.

Dr. Connie Bothwell-Myers, my co-supervisor, your trust and guidance has encouraged me to explore my own ideas. Thank you for your support.

Dr. Maureen Tingley, my co-supervisor, whose knowledge of statistics, true helpfulness, and friendliness have encouraged me since the beginning.

Dr. Wayne Albert, thanks so much for helping during data collection and always being available. You have been so helpful throughout the many stages of this thesis. Your guidance and friendship will not be forgotten.

Dr. Ed Biden, thank you for allowing me to use the San Diego Children's Hospital study's database and the Vicon equipment.

Vicky Chester, thank you for helping create these ideas. Your guidance is appreciated.

The children who participated in this study, your joy for life will never be forgotten. Thank you to all the parents who brought the children to the lab at UNB.

My roomies, it's been a long haul, but now it's done; well, almost, Char. Thanks for being my immediate support line and great friends. I wish you all the best in the years to come. Let's meet up again!

Heather, Adrienne, Kathy, and Mer, each of you in your own way has helped with this thesis. Whether it's a late night session on the phone, or just a place to 
watch some TV, you've all kept me on top of things. You are the best friends I could ever have.

The Atkinson Family, you have been there throughout it all. I do not know of another family who would have been so welcoming or generous. You are truly the kindest people I know. Thank you for being my home away from home.

Barbie, thank you so much for editing this thesis. You put so much of your own time toward this, I don't think I can ever repay you.

My family, I don't know how I can ever thank you properly for the opportunities you have given me. Scott, thanks for bringing me to the Maritimes. I don't think I would have enjoyed this experience as much anywhere else. Mom, you are my best friend; your unselfishness and utter devotion to everything around you is amazing. I can only wish to be like you someday. Dad, your trust and pride in me has given me the courage to continue my education. Maybe I will be the "Doc" someday.

Mark, what can I say? Without you, I would have never had the opportunity to explore these ideas. You have been there each and every day to listen to every possible detail of this thesis. Thank you for being so generous, kind, and loving.

Finally, to all my grandparents, without any of you there would not be any of us. The family that you've created is truly who I am. The memories I share with you will always be a part of me. Whether you are here to see the completion of this work or not, it is to you, Nana, Papa, Granny, and Pomp that I dedicate this thesis. 


\section{TABLE OF CONTENTS}

ABSTRACT.

ACKNOWLEDGEMENTS

ii

TABLE OF CONTENTS

iv

LIST OF TABLES

LIST OF FIGURES

vi

viii

LIST OF APPENDICES.

ix

$\mathrm{x}$

\section{CHAPTER $1:$ INTRODUCTION}

Gait patterns of Children with Developmental Coordination Disorder

Statement of the Problem.

Hypotheses

Significance of the Study

Delimitations.

Limitations.

Assumptions.

Operational Definitions.

\section{CHAPTER 2 : REVIEW OF THE LITERATURE}

Introduction.

Developmental Coordination Disorder (DCD)

Current Areas of Research.

Causes of DCD

Nature of DCD.

Identification of DCD

Instrumentation

Intervention Strategies.

Motor Development in Children.

So What if I Can't Move Well?.

Kinematic Studies.

Gait.

Historical Perspectives and Current Technologies involved in Gait Analysis.

The Gait Cycle.

Gait Patterns of Children

Wilson's One-Dimensional Measure of Normality. 
CHAPTER 3 : METHODOLOGY

Introduction.................................................................................

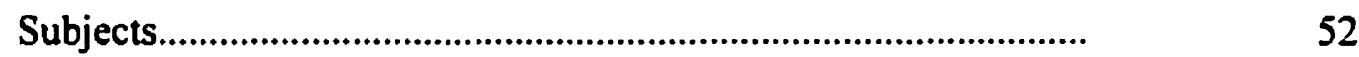

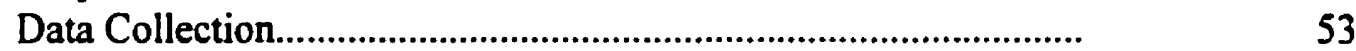

Identification of DCD.......................................................

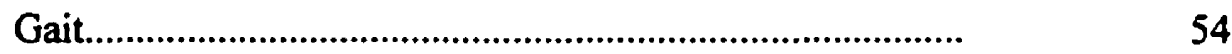

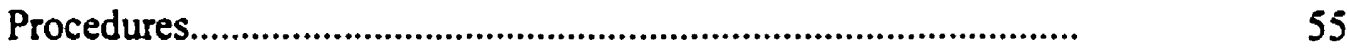

Identification of DCD.....................................................

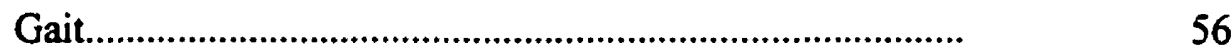

Calculation of the Fscore........................................................................

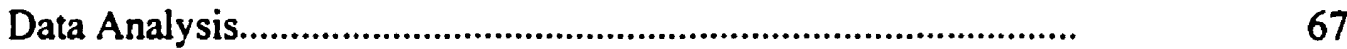

CHAPTER 4 : RESULTS

Screening for Subjects with DCD......................................................

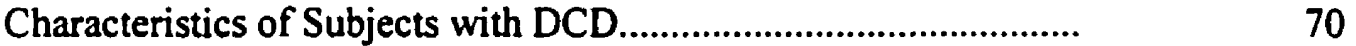

Choosing One Trial per Subjects' Side for Children with DCD.....

Wilson's Score Using Subjects with DCD..............................................

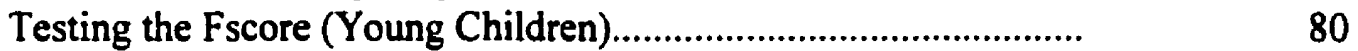

Testing the Fscore (Subjects with DCD)............................................

Results of the Hypotheses Tests..........................................................

CHAPTER 5 : DISCUSSION

Screening for DCD and Subject Characteristics................................

One-Dimensional Measure of Normality Scores.............................

Wilson's (1998) Score.......................................................

Fscore.......................................................................

Subject DCD4 ......................................................................

Subject DCD6............................................................................

Relationship between Children with DCD and Gait Patterns.........

CHAPTER 6 : SUMMARY, CONCLUSIONS, AND FUTURE

RECOMMENDATIONS

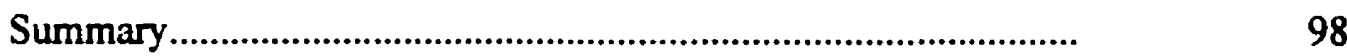

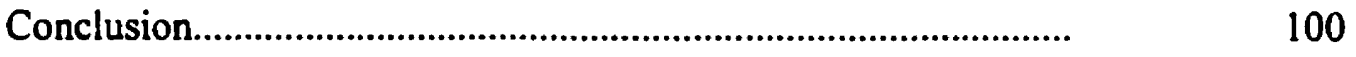

Future Recommendations................................................................

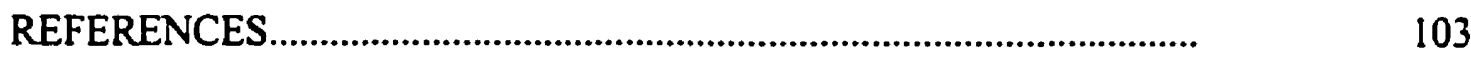

APPENDICES

VITA

vii 


\section{LIST OF TABLES}

Table 3.1 Presence or Absence of a Right Heel Strike, Left Heel Strike, and Reciprocal Arm Swing.

Table 3.2 Percentage of Opposite Foot Strike.

Table 3.3 Mean and Standard Deviation Values of Opposite Toe Off (\%), Single Stance (\%), Toe Off (\%), and Step Length (\%).

Table 3.4 Classification Results of the San Diego Database (three to seven years old) for the Wilson score and the Fscore.

Table 4.1 Results of the Movement ABC for those Subjects Scoring Below the $15^{\text {th }}$ Percentile.

Table 4.2 Results of the DCD Group on the Movement ABC.

Table 4.3 Reported Major Milestones during Early Development of the DCD Group.

Table 4.4 Wilson Scores for Subjects with DCD

Table 4.5 Fscore Classification Results for Children less than Three Years of Age

Table 4.6 Classification Results of the San Diego Database for the Wilson score and the Fscore for Children Less Than Three Years Old

Table 4.7 Gait Characteristics of the DCD group and San Diego Training Set for the Variables Included in the Fscore.

Table 4.8 Gait Characteristics of the DCD group and San Diego Training Set for the Variables Not Included in the Calculation of the Fscore.

Table 4.9 Fscore Classification Results of the DCD Group........................ 


\section{LIST OF FIGURES}

Figure 1.1 Mean Knee Displacement from the San Diego Database............ 5

Figure $2.1 \quad$ Seven-Year-Old Gait.................................................................. 40

Figure 2.2 Summary of the Formulation of the Wilson Score....................... 48

Figure 3.1 Correlation between Percentage of Opposite Toe Off (OTO \%) and Percentage of Single Stance (SS \%)..................................... $\quad 60$

Figure 3.2 Q-Q Plot : Observed Quantiles of $F$ versus the Quantiles

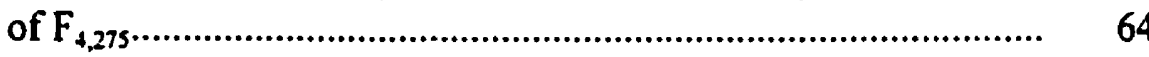

Figure 3.3 Summary of the Formulation of the Fscore.................................... 67

Figure 4.1 Left Hip Displacement of Subject DCD6 and the Mean Hip Displacement Curve....................................................................... $\quad 76$

Figure 4.2 Right Hip Displacement of Subject DCD6 and the Mean Hip Displacement Curve....................................................................

Figure 4.3 Left Hip Displacement of Subject DCD4 and the Mean Hip Displacement Curve.

Figure 4.4 Left Hip Velocity of Subject DCD4 and the Mean Hip Velocity Curve.

Figure 4.5 Left Hip Acceleration of Subject DCD4 and the Mean Hip Acceleration Curve

Figure 4.6 Left Knee Displacement of Subject DCD4 and the Mean Knee Displacement Curve. 


\section{LIST OF APPENDICES}

Appendix A Health/Activities Questionnaire.................................................. 111

Appendix B Gait Laboratory................................................................. $\quad 114$

Appendix C School District 18 Approval........................................................ 116

Appendix D Kinesiology Ethical Approval Form.......................................... 118

Appendix E Letter to Parents and Parental Consent Forms............................ 120

Appendix F Children who were Excluded from the Training Set................... 124

Appendix G Correlation Matrices.................................................................. 126

Appendix H Inverse Covariance Matrices...................................................... 129

Appendix I Missing Data......................................................................... 131

Appendix J Standardized $\underline{B}^{(i)}$ Vectors........................................................... 133

Appendix K Covariance Matrices for the San Diego Database and Children with DCD.. 


\section{CHAPTER 1}

\section{INTRODUETION}

\section{GAIT PATTERNS OF CHILDREN WITH DEVELOPMENTAL COORDINATION DISORDER}

Children place a great deal of importance on being able to move well. Being selected first when choosing teams during any type of play is highly important, not only for social status, but also for physical competency of the child (Bouffard, Watkinson, Thompson, Causgrove Dunn, and Romanow, 1996). Children feel better about themselves if they are viewed as a "good mover" by their peers. But what about the children who do not move well? These children are placed in an uncomfortable situation, as they are looked upon negatively and are regularly excluded from play with other children.

Individuals who are movement incompetent, on the other hand, more often experience negative effects and are less likely to participate in movement situations. Thus, children lacking movement competencies may avoid participation in movement situations (Bouffard et al., 1996, pg 62).

Movement skill acquisition is crucial for enjoying a healthy lifestyle. A healthy lifestyle, including regular physical activity, is being promoted in Canada as it has been shown to reduce the risk of cardiovascular disease, osteoporosis, stroke, and depression (Health Canada, 1999). Creating positive movement experiences throughout childhood will most likely promote an active lifestyle into adulthood. However, negative movement experiences will hinder a child's experiences. That individual will likely refrain from taking part in sports and games as an adult, thus predisposing him or her to 
cardiovascular disease. Children with repeated negative movement experiences will typically avoid physical activity altogether. Although this produces negative physical effects, it can also affect a child psychologically.

Developmental Coordination Disorder (DCD) is defined by the American Psychiatric Association (1994) as having a marked impairment in the development of motor coordination. Diagnosis of DCD is made only when the impairment interferes with academic achievement or daily activities and if the coordination difficulties are not due to any known medical conditions (American Psychiatric Association, 1994). Children with DCD may have difficulties with both fine and gross motor skills. "The term developmental implies that the condition arises during the developmental stage, not that the condition is due to slow development or will it be outgrown" (Fox, University of Western Ontario Web Site).

Studies show that children who do not move well are more introverted and anxious, have fewer social contacts and friendships, participate less in sports during leisure time, have lower academic achievements and ambitions, and fewer social hobbies and pastimes (Cantell, Smyth and Ahonen, 1994; Geuze and Borger, 1993; Schoemaker and Kalverboer, 1994). For these reasons, special attention must be paid to children who have movement difficulties.

Childhood is a time of learning and mastering movement skills. With age, children become more adult-like in their movement patterns. As children mature, their movement patterns become more efficient; new movement patterns emerge from the patterns already mastered. Walking is typically the first adult-like locomotion pattern to 
appear, with many other movement patterns emerging over time such as running, skipping, and jumping. An early study by Shirley (1931) looked at 25 infants from birth to age 2 in order to describe the sequential developmental progression of activities leading to upright posture and a walking gait. She noted that "although the sequence was fixed, individual differences were expressed in variations in the rates of development between infants" (Gallahue and Ozmun, 1989, pg. 167). Walking patterns typically emerge anywhere from 9 to 17 months of age (Gallahue and Ozmun, 1989; Haywood, 1986; Payne and Issacs, 1991). Once walking is mastered, running, skipping, hopping and other advanced movement patterns will emerge (Gallahue and Ozmun, 1989; Haywood, 1986; Payne and Issacs, 1991).

Gait patterns in children have been widely studied. Many of the observable movements have matured by the age of three (Sutherland, Olshen, Biden and Wyatt, 1988) or four years (Whittle, 1991). Cadence, stride length, and walking veloc:ty continue to change with growth, until 15 years of age (Sutherland et al., 1988; Whittle, 1991). Although walking appears to be a very simple task, it is a very complex skill that requires the interaction of the central nervous system, musculoskeletal system, several sensory systems, gravitational forces, and environmental circumstances (Gallahue and Ozmun, 1989). A change to any one of these systems could change the gait pattern of an individual.

Sutherland et al., (1988) attempted to find normative values for gait patterns across children ages one to seven. The study was conducted at the Motion Analysis Laboratory at the Children's Hospital and Health Center in San Diego, California and 
included 413 normal gait observations for 210 males and 205 females over 10 age groups. To ensure the normality classification of the children included in the study, strict criterion were set; the subjects were the product of a full-term pregnancy, born at or after 38 weeks of gestation, walked independently by 14 months, had no orthopaedic problems or treatments, displayed normal growth and development as judged by both parents and family physician, and had not experienced any major medical problems or hospitalizations (Sutherland et al., 1988). The study included lower limb kinematic, kinetic, and electromyographic variables. This look into normative values of gait patterns is extremely important and useful for researchers and clinicians to quantify abnormality and possible warning signals of more serious disorders.

Wilson (1998) used the data from the San Diego Children's Hospital study to investigate a method of quantifying hip, knee, and ankle flexion variability for children aged three to seven. Each joint has a distinctive pattern of travel during the gait cycle. Figure 1.1 shows mean angle displacements from the knee for the normative data by Sutherland et al. (1988). The figure clearly shows the "jerk" each knee performs during a gait cycle. The mean displacement for hip and ankle is much smoother. Observations of a single gait cycle from any child would not give exactly the same pattern as that described by Figure 1.1, due to individual variability. The problem addressed by Wilson (1998) was how to quantify normal, as opposed to abnormal deviation from the mean sagittal hip, knee, and ankle observations. Wilson (1998) recorded the curve of Figure 1.1 as 12 Fourier coefficients (6 coefficients of cosine and 6 coefficients of sine). 


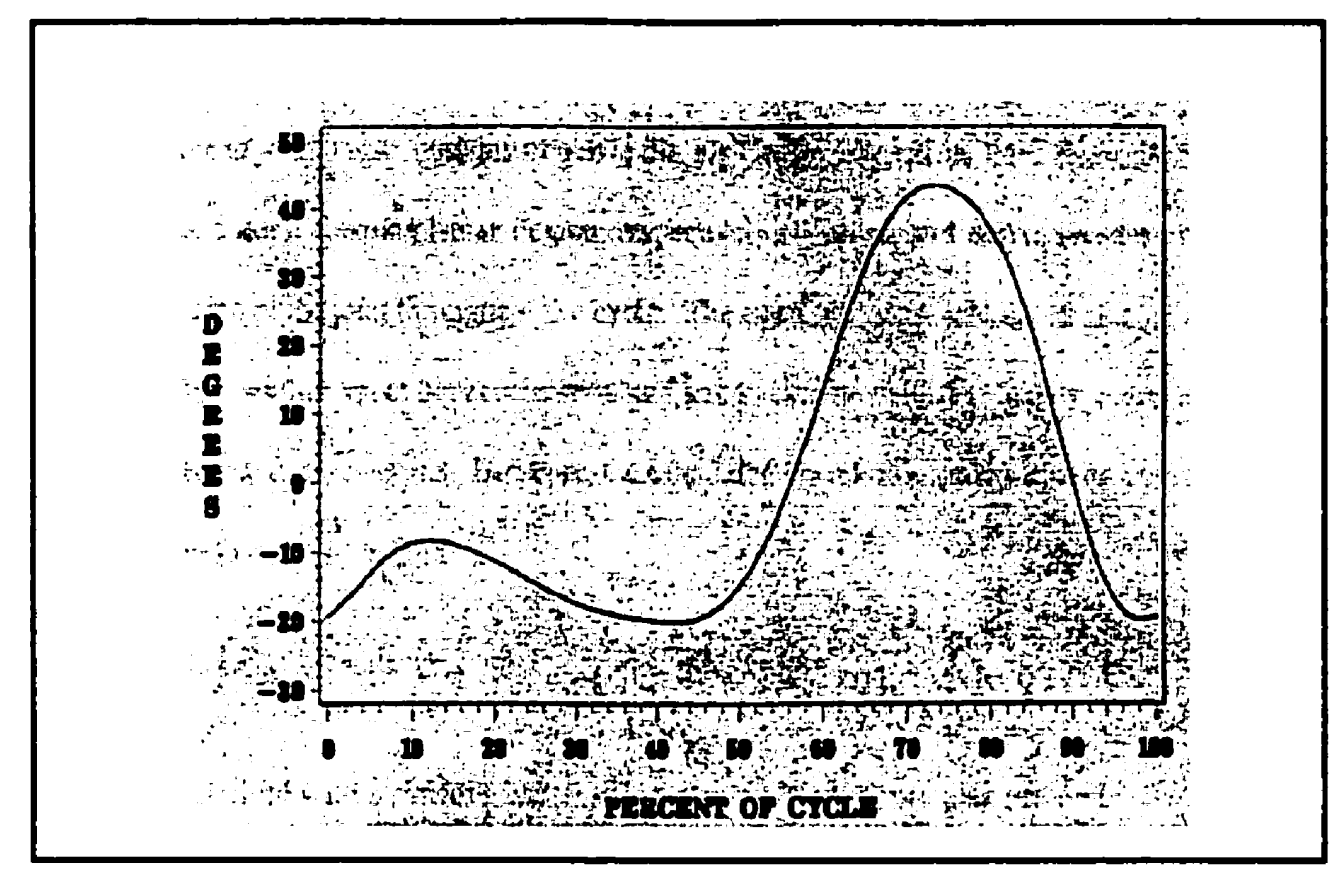

Figure 1.1 - Mean Knee Displacement from the San Diego Database (Sutherland et al., 1988)

Mean hip and ankle flexion curves were also recorded as 12 Fourier coefficients. Thus, mean or average gait patterns were recorded as a 36-dimensional observation. Wilson (1998) also recorded covariance structures of individual variability corresponding to this 36-dimensional mean. Finally, Wilson (1998) developed a one-dimensional measure of normality of gait which is calculated by comparing a child's gait pattern with the mean patterns (such as Figure 1.1), while allowing for correlations between hip, knee, and ankle displacement, velocity, acceleration, and primary frequency. Deviation from the mean joint rotation curve allows for diagnostic interpretation of abnormality. The normative one-dimensional statistic was then tested using very young children and 
children up to seven years of age who were born prematurely. This type of classification discriminated well between normal and abnormal gait patterns.

Wilson (1998) only used sagittal hip, knee, and ankle kinematics, which may be difficult for clinicians to use as they typically do not have the necessary equipment to record biomechanical data. Therefore, the time/distance variables such as cadence, stride length, and the percentage of toe off, may be more appropriate, as these measures are typically more often used and easier to record.

Children with DCD typically have problems with gross motor skills such as running, jumping, and kicking. Therefore, looking at a movement pattern typically mastered before these movement skills could prove to be of value for identifying children with DCD. Walking is a cyclical, everyday movement that goes unappreciated until it is impaired by disease or injury.

Walking is needed for certain test items of the Movement $A B C$, which is frequently used as a diagnostic instrument for DCD (Henderson and Sugden, 1992). The children included in the present study were assessed using the Movement $A B C$ Test. Both Age Band I ( 4 to 6 years) and II ( 7 to 8 years) have a walking component included in the test. Age Band I requires children to walk a 4.5 metre line (15 steps) with the heels raised, while Age Band II requires children to walk along the same 4.5 metre line, while placing one foot against the toe of the other. Although DCD cannot be diagnosed from failure of this component alone, it may identify a child as having a severe motor impairment rather than a minor impairment. 
Current literature suggests little in the way of walking problems for children with DCD, however the definition of DCD by the American Psychiatric Association (1994) stipulates that a diagnosis only occurs if the problem of movement skill interferes with daily activity. As walking is a daily activity and a basic movement pattern, it could be a skill that children with DCD have problems mastering. Revie and Larkin (1993) suggest that children who "look awkward" and are described as clumsy, dyspraxic, or poorly coordinated have difficulty performing basic movement patterns such as walking, running, hopping, jumping, throwing, catching, kicking, and hitting.

\section{STATEMENT OF THE PROBLEM}

The purpose of this study was to validate Wilson's (1998) statistical procedure on a population that may not be classified as abnormal by naked eye observation. Wilson's one-dimensional measure of normal gait is based on sagittal hip, knee, and ankle flexion/extension data. One might suspect that for children with DCD, clinical gait differences are not as apparent as for children with Down's Syndrome or Hypotonia, due to the nature of the disorder. A secondary objective of this project was to formulate another one-dimensional measure of normal gait using the time/distance variables, as these variables are measured more frequently using inexpensive methodologies. Therefore, by formulating a one-dimensional measure of normality using the time/distance variables, clinicians and other educators will have a more readily accessible technique for diagnosis of DCD. 
In order to compare gait differences of children with DCD with those who are classified as normal, angular movement patterns from the sagittal view of the hip, knee, and ankle were obtained. Using the one-dimensional measure of normality developed by Wilson (1998), children with DCD were classified as normal, abnormal, or unusual.

Time/distance variables (cadence, stride length, cycle time, walking speed, percentage of toe off, percentage of opposite foot strike, percentage of opposite toe off, and percentage of single stance) were also recorded for each subject. Examination of the correlation matrix for the normative database was done in order to identify which time/distance variables were predictable from others and which variables appear to be independent. Following Wilson's (1998) methodology, a one-dimensional measure of normal gait was formed. Children with DCD were then analyzed as normal, abnormal, or unusual according to this new time/distance measure. Mean and Covariance structures of the time/distance variables was compared for children with DCD and the normative San Diego database.

\section{HYPOTHESES}

One migint expect that children with DCD would be classified as abnormal using Wilson's (1998) statistical procedure. One might have also hoped that children with DCD would differ systematically from children's normal sagittal hip, knee, and ankle gait patterns so that they would be classified as a homogenous group. Expectations were such that children with DCD would differ from children classified as normal on the time/distance variables again. Finally, children with DCD were expected to be classified 
as abnormal, using the one-dimensional measure of normality for the time/distance variables. Therefore, this study testing the following three hypotheses:

1. The one-dimensional measure of normality for the sagittal hip, knee, and ankle curves by Wilson (1998) would classify most children with DCD as abnormal.

2. a. There would be differences in the means between normal children and children with DCD, using the time/distance variables.

b. There would be differences in the covariance structure between normal children and children with DCD, using the time/distance variables.

3. The new one-dimensional measure of normality based on time/distance variables developed in this thesis would classify most children with DCD as abnormal.

\section{SIGNIFICANCE OF THE STUDY}

This study was significant for its kinematic descriptions about gait patterns of children with $\mathrm{DCD}$. Not only did this add to the relatively sparse information regarding $D C D$, but it also provided insight for the development of new diagnostic or intervention strategies. An earlier belief that children with DCD will "grow out of it" has been contradicted by recent research (Cantell et al., 1994; Geuze and Borger, 1993; Losse, Henderson, Elliman, Hall, Knight and Jongmans, 1991). A look at children originally diagnosed at an early age and retested in adolescence, showed that among the severe cases, DCD is still prevalent in adolescence and those afflicted showed signs of maladjustment (Cantell et al., 1994; Geuze and Borger, 1993; Losse et al., 1991). 
Gait analysis is extremely difficult because there is an abundance of data produced by a single gait cycle, yet researchers have not come up with a common method of analyzing large data sets. Wilson (1998) attempted to find a simple, one-dimensional score that classified children's sagittal hip, knee, and ankle angle patterns as normal, abnormal, or unusual. Wilson's (1998) study used the children who participated in the normative study at the San Diego Children's Hospital (Sutherland et al., 1988). The procedure was then tested using very young children and children up to the age of seven years who were born prematurely and found it discriminated well between normal and abnormal gait patterns. Using children with DCD, whose gait patterns were of interest and possibly abnormal, to determine if this method of classification is beneficial to other researchers and clinicians was of interest. Wilson's one-dimensional measure of normal gait used only sagittal hip, knee, and ankle flexion/extension curves and therefore, it was of interest to determine if other variables such as the time/distance measures would be of the same value, especially to clinicians and educators.

The basic knowledge of gait patterns of children with DCD benefits researchers and clinicians working with these children. The one-dimensional measure of normal gait was simply formulated to determine its usage for people attempting to classify children's gait as normal or abnormal.

\section{DELIMITATIONS}

1. The subjects included in the DCD group for this study were limited to grade 1 students from School District 18, in Fredericton, New Brunswick. The control 
group was limited to the children who took part in the normative gait study in San Diego, California at the Children's Hospital in 1988. The DCD group was comprised of children whose score was at or below the $15^{\text {th }}$ percentile on the MABC Checklist (Henderson and Sugden, 1992), as well as at or below the $15^{\text {th }}$ percentile on the MABC Test (Henderson and Sugden, 1992).

2. The assessment of fine and gross motor ability was limited to the use of the MABC Checklist and Test (Henderson and Sugden, 1992).

\section{LIMITATIONS}

1. Children's motivation and attitude during testing and gait analysis may have contributed to performance outcomes.

2. Children within the DCD group were assigned to an age bracket for the diagnostic testing (Movement $\mathrm{ABC}$ ) as of their last birthday. The children in the San Diego Children's Hospital study were plus or minus one month of their birthdays on the date of testing.

3. The samples of both children with DCD and the children classified as normal from the San Diego database were not random, but self-selecting volunteers.

\section{ASSUMPTIONS}

1. Scoring objectivity and consistency were maintained by having one tester administer the same tests to all of the children throughout this project. 
2. Children received equal amounts of instruction during each phase of this experiment.

\section{OPERATIONAL DEFINITIONS}

1. Developmental Coordination Disorder: refers to children having a marked impairment in the development of motor coordination. Diagnosis of DCD is only made when the impairment interferes with academic achievement or daily activities, and if the coordination difficulties are not due to any known medical conditions (APA, 1994).

2. San Diego database: refers to the data that were produced from the study done by Sutherland et al., at the San Diego Children's Hospital in 1988.

3. San Diego training set: refers to the group of children that were included, from the San Diego database, in the calculation of the Wilson score and the Fscore

4. UNB: refers to the University of New Brunswick.

5. Gait cycle: refers to movements and events that occur between successive footsteps of the same foot (Sutherland et al., 1988, pg. 16).

6. Cadence: refers to the number of steps per minute (Sutherland et al., 1988, pg. 16).

7. Stride length: refers to the distance traveled by the same point on the same foot during two successive steps. Each stride length comprises of one right and one left step length (Sutherland et al., 1988, pg. 16). 
8. Normal gait pattern: refers to having an interpretable Wilson score less than 1.73 (Wilson, 1998, pg. 76).

9. Abnormal gait pattern: refers to having an interpretable Wilson score larger than 2.31 (Wilson, 1998, pg. 76).

10. Unusual gait pattern: refers to having an interpretable Wilson score falling between 1.73 and 2.31 (Wilson, 1998, pg. 76). 


\section{CHAPTER 2}

\section{REVIEW OF LITERATURE}

\section{INTRODUCTION}

This literature search and review contains relevant information in the areas of DCD, normal growth and child development, the importance of moving well, kinematic studies of children who do not move well, gait patterns of children, and Wilson's (1998) statistical procedure and methodology.

\section{DEVELOPMENTAL COCRDINATION DISORDER}

Active living is popular among many individuals in today's society. One dimension of a healthy and active lifestyle includes regular physical activity. Individuals who do not move well are placed in an uncomfortable situation because they are faced with doing something that they are not able to perform with complete confidence. If individuals are not comfortable doing something, they will be more inclined to refrain, thereby leaving themselves at risk for an inactive lifestyle, which may lead to obesity and heart disease (Health Canada, 1999). Childhood is a time when movement patterns are learned and mastered, and therefore, where patterns of physical activity emerge. Thus, researchers are becoming increasingly concerned with investigating children with Developmental Coordination Disorder (DCD). 
The Adapted Physical Activity Quarterly (Human Kinetics, 1994, vol. 11) and Human Movement Science (Elsevier Science, 1998) have each committed an entire volume to research on children with DCD. Articles within these two volumes cover a wide range of topics such as identification tools, possible causes of DCD, and intervention strategies.

Developmental Coordination Disorder (DCD) is defined by the American Psychological Association as a marked impairment in the development of motor coordination in children. Diagnosis of DCD is made only when the impairment interferes with academic achievement or daily activities and if the coordination difficulties are not due to any known medical conditions (American Psychiatric Association Diagnostic and Statistical Manual IV, 1994). "The term developmental implies that the condition arises during the developmental period, not that the condition is due to slow development or will be outgrown" (Fox, University of Western Ontario Website). DCD occurs in approximately $5 \%$ to $16 \%$ of children (Henderson and Hall, 1982; Wright, Sugden, $\mathrm{Ng}$ and Tan, 1994), with the Diagnostic and Statistical Manual IV estimating that $6 \%$ of school age children between ages 5 and 11 have DCD (APA, 1994).

Identifying children with Developmental Coordination Disorder is relatively difficult, due to the lack of agreement in terminology and diagnostic protocol amongst researchers. An example of such inconsistency is that Developmental Coordination Disorder is used as a heading by the American Psychiatric Association (DSM-IV, 1994), while the World Health Organization has used Specific Developmental Disorder of Motor Function (Henderson, 1994). Labels such as "clumsy" or "clumsiness" (Dare and 
Gordon, 1970; Fox and Lent, 1996; Henderson and Hall, 1982; Henderson, 1987; Knuckey and Gubbay, 1983; Lord and Hulme, 1987; Reuben and Bakwin, 1968; Schoemaker, Hijlkema and Kalverboer, 1994; Skorji and McKenzie, 1997), "developmentally delayed" (Majnemer and Shevell, 1995), "physically awkward" (Causgrove Dunn and Watkinson, 1996), "developmental motor deficits" (Dewey and Kaplan, 1992), and "motor coordination problems" (Maeland, 1992) have been used throughout the literature. However, Developmental Coordination Disorder is starting to be used more frequently (Henderson, Rose and Henderson, 1992; Hoare, 1994; Piek and Edwards, 1997; Smyth and Mason, 1997; Wright, 1997; Wilson and McKenzie, 1998; Wright and Sugden, $1996 \mathrm{~b}$ ). The lack of agreement regarding terminology poses a serious threat to researchers trying to study this young group of individuals, as they may not receive all relevant information due to the difference in vocabulary.

Although there is definitely a growing interest in $D C D$, only a small number of researchers have chosen to look at the kinematics of children with DCD. A biomechanical analysis of gait patterns of children with DCD would provide insight into the proper identification and development of intervention strategies. Two studies reported the usefulness of a biomechanical analysis when working with children with movement difficulties (Hsu, Bardfield, Cratty, and Garfinkel, 1987; Marchiori, Wall, and Bedingfield, 1989). The next few pages include a further investigation of children with DCD. 


\section{CURRENT AREAS OF RESEARCH}

The current research performed on children with Developmental Coordination Disorder (DCD) can be classified into several different areas of study: the possible cause of DCD, the nature of $D C D$, identification of children with $D C D$, psychological and social aspects of DCD, and intervention strategies.

\section{Causes of DCD}

One area of research addresses the possible causes of DCD. A recent study by Wilson and McKenzie (1998) performed a meta-analysis to identify processing operations most strongly associated with motor impairment. Their findings indicate that perceptual problems, particularly visual-spatial, were associated with problems of motor coordination. This is in agreement with Lord and Hulme (1987), who have suggested that $\mathrm{DCD}$ could be related to a deficit in visual-perception processing. Others (Laszlo and colleagues; Piek and Coleman-Carman, 1995) have indicated that a deficit lies in kinesthetic-perceptual processing. Skorji and McKenzie (1997) also supported the finding that the deficit lies within the visuospatial processing, as children with DCD had a harder time recalling a movement after a high visual-spatial interference was used. Smyth and Mason (1997) put a group of children with DCD and a control group (age and ability matched subjects) through three proprioceptive tasks. They concluded that children with DCD have a lack of proprioception, however, they can plan a movement normally. Other possible causes could be linked with the rate at which these children learn a new movement skill (Missiuna, 1994). 


\section{Nature of DCD}

Along with the causes of DCD, the nature of DCD has been studied. Hoare (1994) made the first attempt to form subgroups within a diagnosed group of children with DCD. Five sub-clusters of children were present with similar motor difficulties. However, Wright and Sugden (1996 a) also investigated the nature of inter- and intra-group differences and found four sub-clusters of DCD. The lack of homogeneity within a group of children diagnosed with DCD poses a threat of external validity to the research that is and has been conducted on these children. The medical profession has also taken a strong interest in studying children with DCD. Although no known medical condition is present in children with DCD, as indicated by the APA definition (1994), the medical profession seems to focus on DCD as a neurological disorder (Fox and Lent, 1996; Jacobson, 1998), or classifies DCD as "minimal cerebral damage" (Reuben and Bakwin, 1968). In the above cases, the articles used "clumsiness" instead of referring to DCD, therefore researchers cannot be sure they are considering the same group of children.

\section{Identification of DCD}

Identification of DCD is difficult because of the lack of agreement amongst researchers' diagnostic protocols. Several motor development tests have been developed to aid in the identification and screening process for children with DCD. Family or school board physicians, parents, teachers, and researchers are the people most likely to identify children with DCD. 
Identification of DCD can be done by family and school board physicians. If a parent or guardian notices something abnormal with a child, the physician will be the first person the parent or guardian contacts. One research group (Schoemaker et al., 1994) used a medical examination as part of the screening process for subject selection. The medical examination was performed to search for pathology and to evaluate the sensory and motor systems (Schoemaker et al., 1994). However, further tests were done on those students who "passed" the medical examination ensuring there was no known medical condition.

In some cases, the child's teacher brings the developmental disorder to the attention of the parents. Teachers, who spend the greater part of the day with their students, assist researchers in the identification of children who might be considered to have DCD. Piek and Edwards (1997) investigated the difference between physical education teachers and classroom teachers in their ability to detect DCD. Classroom teachers were able to identify only $25 \%$ of children with DCD, while physical education teachers were able to identify $49 \%$ of the children who were diagnosed using the MABC Test. Similar results were found by Maeland (1992), who reported that classroom teachers had difficulty identifying children with DCD compared to the identification with the Test of Motor Impairment and the Test of Motor Proficiency. However, Henderson and Hall (1982) found a high correlation between teachers' judgements and identifying children with DCD using a neurodevelopmental examination, the Motor Impairment Test, the Wechsler Intelligence Scale for Children, and the Schonell Reading Test. 
The reason for controversial findings may lie in the age of the subjects, the training of the teachers, the time of year the research took place, and the instrumentation used by the teachers. Subjects in the Henderson and Hall (1982) study were younger (6 years of age) as compared to Maeland (1992) who used subjects who were older (10 years of age). With increasing age, children at school spend more time in the classroom with therefore less time for the teachers to watch them as they move about (Maeland, 1992). Most likely, by 10 years of age, the classroom teacher and physical education teacher will be different. Training of the teacher could have an effect on the results because physical education teachers were able to identify more children than classroom teachers (Piek and Edwards, 1997). For a year prior to the study conducted by Henderson and Hall (1982) teachers were trained to ensure that teachers knew what they were looking for in children they viewed as "clumsy" and those classified as "normal." Therefore, making teachers more aware of the problem by educating them as to what is considered "clumsy" and "normal" motor development, would certainly increase the chance that the child with DCD could be properly identified. The time of year the study was conducted may also have an effect on the diagnostic capabilities of teachers. Early in the school year, teachers will not be all that familiar with each student. Therefore, asking teachers to nominate children in their classroom close to the end of the school year would produce more accurate results. Finally, the instrument used to nominate children who may have DCD must have an effect on the results. The instrument used must be easy for the teacher to use. The following section describes the current instruments available to researchers and clinicians. 


\section{Instrumentation}

There are two basic types of assessment instruments: normative-referenced and criterionreferenced assessments (Payne and Issacs, 1991). Normative-referenced assessments are quantitative in nature and compare an individual's performance to others of similar age, gender, and socio-economic status (Payne and Issacs, 1991). These types of tests are relatively easy to administer, minimal training is required by the administrator, and scoring procedures are simple. However, normative-referenced assessments cannot specify the exact problem or developmental deficit; they simply give overall information about how an individual relates to others of his or her similar background. On the other hand. "criterion-referenced assessments can evaluate the 'quality' of a person's performance" (Payne and Isaacs, 1991, pg. 329). Motor development has a known sequence of milestones, so criterion-referenced assessments seek to find where in the developmental milestones the individual ranks. This type of assessment can also be referred to as "process-oriented" (Payne and Isaacs, 1991), as it compares performance along a continuum from immature to mature movement patterns. However, with this type of testing, more training is needed by the administrator than when using normativereferenced assessments.

Many researchers have used normative-referenced assessment tools because of the relatively simple method of administering and scoring the test. Normative-referenced tests pose somewhat of a problem to researchers and clinicians alike. The arbitrary cutoff points are not well established or agreed upon in different countries. Ulrich (1985) points out that most of the assessment tools are unpublished or lack standardization. 
Many of the published tests measure the product of motor performance in terms of time, distance, or accuracy and if the test indicates that a child is deficient, it is difficult to recognize the underlying motor deficits. Also, with the published tests, most offer a normative-referenced interpretation exclusively and provide no information useful for instructional programming. The normative-referenced tests rely solely on product scores, focus on the abilities of the child compared to what they cannot do, and judge children against a chronological age (Ulrich, 1985).

Identifying children with Developmental Coordination Disorder is relatively difficult because of the lack of agreement in diagnostic tools. Tools vary depending on geographic area as well as the academic background of the researcher. Several identification tools have been constructed and used frequently throughout the literature. The most common tools that researchers have used were the McCarron Assessment of Neuromuscular Development (Armitage and Larkin, 1993; Hoare, 1994; O’Beirne, Larkin and Cable, 1994), the Test of Motor Impairment (Henderson et al., 1992; Maeland, 1992; Skorji and McKenzie, 1997), the Movement ABC which has succeeded the Test of Motor Impairment (Piek and Coleman-Carman, 1995; Smyth and Mason, 1997; Wright and Sugden, 1996 a, b) and the Motor Performance Test Battery and Canada's Fitness Test (Marchiori et al., 1987). As well, most children have been initially nominated by a teacher who has filled out some type of questionnaire about the child (Maeland, 1992; Missiuna, 1994; Skorji and McKenzie, 1997; Wright and Sugden, 1996 b). Researchers need to be concise with respect to the protocol for identifying children with DCD because cut-off points are not consistent between tests, which results in a large 
variability in the prevalence and severity of DCD. The MABC seems to be the "Gold Standard" for diagnosing children with DCD, and is further described below.

The Test of Motor Impairment (TOMI) was originally devised by Stott, Moyes, and Henderson (1972) to provide information on motor deficiency (Riggen, Ulrich, and Ozmun, 1990) and again revised by Stott, Moyes, and Henderson in 1984 . The first revision of the TOMI (TOMI-Henderson Revision in 1984) included eight items divided into manual dexterity, static and dynamic balance, and ball skills. The test included a relatively small number of performance items that were assumed to be fundamental to motor skill development (Causgrove Dunn and Watkinson, 1996). The TOMI-Henderson Revision attempted to use "culturally-normal" subtests. However, research performed by Causgrove Dunn and Watkinson (1996) revealed that the TOMI-Henderson Revision is gender-biased in favour of males. Seventy-four percent of females would not have been diagnosed with DCD had they passed the ball skills section of the Test. The $74 \%$ includes 35 of 36 females who were diagnosed with moderate DCD. Therefore, only one female would have been diagnosed as having moderate DCD if she had passed the ball skills test or had those items been eliminated.

The problem with gender bias in identification and screening tools is that researchers need to be able to fairly assess both males and females. Skorji and McKenzie (1997) used the TOMI-Henderson Revision as one of the screening tools for their study and found 20 subjects, 14 male and 6 female, who were classified as "clumsy." Similar findings were found by Maeland (1992) who first used the Test of Motor Proficiency (TMP) and then the TOMI-Henderson Revision on the subjects diagnosed as "clumsy" by 
the TMP. Out of a sample size of 221 children, 10 boys and 4 girls were identified by the TOMI-Henderson Revision as having severe motor difficulties. These studies suggest that the TOMI-Henderson Revision (1984) has done well at assessing females and presents contradictory evidence to the study done by Causgrove Dunn and Watkinson (1996).

The contradictory results could be explained by the fact that the items on the TOMI were "culturally learned" items. Different cultures place different amounts of importance on learning certain movement patterns. The females identified by Skorji and McKenzie (1997) and Maeland (1992) could have had more exposure to ball skills. As well, these differences suggest a problem that children diagnosed with DCD may merely have had limited exposure to the activity that was being tested. Normative-based identification tools present the problem of being unable to distinguish between a lack of exposure or to correctly identify the intrinsic factor which makes these children uncoordinated.

Henderson and Sugden (1992) have revised the Test of Motor Impairment which is now known as the Movement Assessment Battery for Children (MABC). The MABC contains three parts: the MABC Checklist, the MABC Test, and guidelines for remediation (Wright et al., 1994). The MABC is recommended to researchers as a screening tool and to practitioners as a diagnostic tool (Henderson and Sugden, 1992). The first part of the Movement ABC package is the Checklist (Henderson and Sugden, 1992). The first MABC Checklist, a criterion-referenced test, was devised by Sugden (1972) with revisions by Sugden and Sugden (1991) and again revised for the Movement 
ABC package (1992) (Wright et al., 1994). The MABC Checklist is designed mainly for primary school teachers to aid in the identification and management of skill problems because it looks at the relationship that the children have with the environment. The MABC Checklist provides information regarding the nature of the developmental problem, as the Checklist is divided into several different sections: the child stationary/environment stable, child moving/environment stable, child stationary/environment changing, child moving/environment moving, and behavioural problems related to motor difficulties.

The MABC Test is a normative-referenced assessment tool and consists of three sections: manual dexterity, ball skills, and static and dynamic balance, similar to the TOMI. Also, the MABC Test is organized for different age bands: Age Band I is 4 to 6 years old, Age Band II is 7 and 8 years old, Age Band III is 9 and 10 years old, and Age Band IV is 11 and 12 years old (Henderson and Sugden, 1992).

The Movement ABC (Henderson and Sugden, 1992) is a reliable and valid test. According to the Test manual, the total impairment scores of the MABC Test have shown a $97 \%$ agreement for age 5 and a $91 \%$ agreement for age 7 after a two-week reassessment of the same children (Henderson and Sugden, 1992). The MABC Test has been validated against the Bruininks-Oseretsky Test. The total impairment scores were subdivided into four categories and then the score on the Bruininks-Oseretsky test was compared to those scores. Results indicated that the Bruininks-Oseretsky test scores of the lowest scoring Movement $\mathrm{ABC}$ group differed significantly from the other three groups (Henderson and Sugden, 1992). Reliability of the MABC Checklist was studied 
by readministering the Checklist after a one-month period. A correlation coefficient of 0.89 was found to be significant $(p<0.001)$. The validity of the MABC Checklist was studied using the 1984 edition. The validity of the MABC Checklist investigated the relationship between the MABC Checklist and the MABC Test. Results indicated that children with a motor impairment and those who were classified as normal were identified using this Test and Checklist (Henderson and Sugden, 1992). As well, another measure was done to ensure that the Checklist and Test identified the same children as motorically impaired and normal. Researchers looked at the number of children identified by both the Checklist and Test and found that 7 of 16 children had different identifying scores. This indicates that teachers using the Checklist generally identify more children than the MABC Test.

The problem with the MABC is that it has not yet been validated to determine if a gender bias still exists, as in the TOMI (Causgrove Dunn and Watkinson, 1796). The MABC Checklist and Test have been used by many researchers (Piek and ColemanCarman, 1995; Smyth and Mason, 1997; Wright and Sugden, 1996 a, b). Researchers from Holland (Smits-Engelsman, Henderson and Michels, 1998), Sweden (Rosblad and Gard, 1998), Japan (Miyahara, Tsujii, Hanai, Jongmans, Barnett, Henderson, Hori, Nakanishi and Kageyama, 1998), and Singapore (Wright et al., 1994) are investigating the usefulness of the MABC within their culture. Preliminary research suggests that only minor alterations may be necessary.

Wright and Sugden (1996 b) have suggested the need to address the issue of identification rather than debating which test is more appropriate. In order to assess 
whether a child meets the criteria of diagnosis by the DSM-IV (1994) definition, a normative-referenced test is needed to remove any debate of a serious motor impairment and a criterion-referenced test is needed to address problems that the child might have with daily living (Wright and Sugden, 1996 b). Missiuna and Pollock (1995) also point out the need for multiple sources of information when attempting to identify children with developmental probiems:

A comprehensive assessment should include consideration of the interplay between the child and the environment and a contextual approach to assessment should be used to create a frame of reference for the interpretation of data collected (Missiuna and Pollack, 1995, pg. 58).

Therefore, researchers need to use more than a single test to properly identify children with DCD. The MABC provides both criterion-referenced and normative-referenced tests, as multiple sources of information.

\section{Intervention Strategies}

Another research approach to DCD research has focused on intervention strategies. Due to the diverse nature of $\mathrm{DCD}$, intervention strategies must examine the child directly and address his or her individual problem(s) (Hoare, 1994). After children see their family physician or pediatrician, doctors normally refer those with DCD to a physiotherapist or occupational therapist, speech pathologist, psychologist, or a special educator (University of Western Ontario Web Site, "Developmental Coordination Disorder: Twenty Questions"). 
A recent review article by Sigmundsson, Pedersen, Whitting and Ingvaldsen (1998) reported different approaches to helping a child with DCD: perceptual motor training, sensory integration therapy, kinaesthetic training, cognitive affective training, physiotherapy, and task-specific intervention. Perceptual motor training looks at the relationship between perceptual qualities and motor abilities. Sensory integration therapy is based on the ability to organize sensory information, thereby improving academic and motor skills. Kinaesthetic training concentrates on improving kinaesthetic or proprioceptive awareness in order to generalize to motor performance. Physiotherapy, as a means of intervention, stems from the premise that various abilities underlie specific movement patterns. Developing a repertoire of basic movement abilities should increase overall motor ability. Schoemaker et al. (1994) investigated the role of a physiotherapy intervention for a three-month period. Testing the children after three months of a physiotherapy plan, followed by three months of no intervention still favoured the physiotherapy program. However, Schoemaker et al. (1994) also pointed out that the favourable results could have stemmed from building confidence to perform the movement skills rather than the actual physiotherapy program. Finally, task-specific intervention includes teaching the child to move within a specific environment, which should aid for that specific movement. The review article by Sigmundsson et al., (1998) presents a good overview of many of the intervention strategies presently being employed. However, other intervention strategies do exist and are currently being validated. 
Other researchers have attempted to give children with DCD a movement education program, which in effect, gets the child moving in any way possible (Bischoff and Lewis, 1987; Caine, 1990). The UNB Motor Development Clinic chose activities that were directly related to the tasks on the Test of Gross Motor Development (Ulrich, 1985), which is the test originally chosen for diagnosis of DCD (Caine, 1990). However, Bischoff and Lewis (1987) looked at a movement education program where "the content of educational games, dance, and gymnastics was developed and taught through the themes of space, effort, body, and relationship awareness" (Bischoff and Lewis, 1987, pg. 348). The purpose of the Bischoff and Lewis (1987) study was to evaluate fitness levels of the children taking part in the movement education program. Researchers indicated that children in the movement education program needed remediation in cardiovascular fitness and a reduction of subcutaneous fat. Bischoff and Lewis (1987) did not believe that this movement education program was fulfilling the purpose originally stated.

Dowsett (1995) has recently reported on the work of Polatajko, called "verbal self-guidance" (VSG). The child talks his or her way through the task, breaking it down into simple steps while providing self-encouragement to him or herself for each step forward. Polatajko indicated that children have a different outlook on their movement skills, and have displayed more of a willingness to attempt new things (Dowsett, 1995). Martini and Palatajko (1998) reported that different clinicians could achieve similar results using the treatment approach, VSG. The research by Martini and Palatajko (1998) have provided further evidence that VSG is an effective intervention approach. 
The various intervention strategies may be somewhat different in theory, however, they all focus on getting the child moving. Researchers and clinicians have to be skeptical about their treatments because, as Fox points out, "children can succeed with certain charismatic clinicians and people wrongly attribute the source of the success to the program, instead of the individual" (Dowsett, 1995, pg. 21). Howard (1997) reported that there is no available instrument that allows clinicians to measure the importance of intervention programs. It is not feasible to administer the original diagnostic instrument after short intervention programs because of the transfer effect of testing. Therefore, children with DCD need an intervention strategy that can measure improvements over time and that does not involve a transfer effect of testing.

A recent report by Leemrijse, Meijer, Vermeer, Lambregts and Ader (1999) investigated the individual change in children with mild to moderate motor impairment using the MABC Test. Results indicated that the total impairment score was sensitive enough to monitor individual change, while the cluster scores had a moderate sensitivity and the individual test items were not appropriate to detect individual change. However, time was interpreted as a significant effect of learning. Researchers and clinicians must have a full understanding of DCD and the intervention strategy used, in order to claim an improvement in motor performance. Therefore, in order to understand children with DCD, researchers and clinicians must have a strong basis of normal motor development. 


\section{MOTOR DEVELOPMENT IN CHILDREN}

Normal motor development has a typical pattern which it follows, however, the onset occurs at different times for the individual child. As a child matures, his or her movement patterns become more efficient, and new movement patterns emerge from the older ones. With age, children become more adult-like in their movement patterns.

Gallahue and Ozmun (1989) suggested that walking occurs at approximately 13 months; running, jumping, and kicking at 18 months; hopping at 3 years; and galloping and skipping at 4 years of age. Haywood (1986) indicated that walking can occur anywhere from 9 to 17 months of age and by age 2 the child will have most of the essential ingredients of an advanced walker. Also suggested by Haywood (1986), running and the early stages of jumping occur around 2 years of age; repeated hopping will not be seen before 3 years of age and can last until at least the child is at least 5 years old; galloping will be seen once running has occurred and before hopping can be mastered; and finally, sliding and skipping can be seen anywhere between 4 and 7 years of age. Payne and Issacs (1991) also suggested similar findings, that independent walking is apparent by 12 months of age, even though the norm is considered from 9 to 17 months. The authors suggested that running will occur somewhere between 18 and 24 months and that jumping (including hopping and leaping) will be seen once the child can run. Normal progression of child development moves from walking independently to further movement patterns of running, hopping, galloping, and leaping. If a problem occurs anywhere along the pattern of development, it is assumed that the emergent patterns would also be affected. As children place such a high importance on being able 
to move well (Bouffard et al., 1996), children who do not move well have shown negative effects in the social and emotional domains.

\section{SO WHAT IF I CAN'T MOVE WELL?}

Why is it so important for children to be able to move well? Many of the longitudinal studies (Cantell et al., 1994; Geuze and Borger, 1993; Losse et al., 1991) stress the fact that intervention strategies do help children with DCD alleviate some of their problems (Wright, 1997). An earlier belief that children with DCD "grow out of it" and will improve with maturation (Wright, 1997) has been discredited by many of the longitudinal studies (Cantell et al., 1994; Geuze and Borger, 1993; Losse, et al., 1991).

Children with DCD tend to have social and emotional problems demonstrated by being more introverted, judging themselves less physically and socially competent, and being generally more anxious than their peers (Schoemaker and Kalveroboer, 1994). Geuze and Borger (1993) looked at the prevalence of DCD five years after the original diagnosis. Only one-half of the original study participants were available, however, results indicated that one-quarter of children originally diagnosed with DCD had improved. Yet, the other quarter of children that still had movement difficulties had general characteristics of having to repeat a grade in school, had fewer social contacts and friendships, and reported less participation in sports activities during leisure time.

As well, Cantell et al. (1994) have shown that five years after being diagnosed, children with DCD had fewer social hobbies and pastimes and lower academic achievements and ambitions, even though they did not have a poor opinion of their social 
acceptance or self-worth. The intermediate group in this study, consisting of the children who did grow out of it, had good school performance, high ambitions, and engaged in social sports. Children need to be able to participate with their peers in activities in order to grow and mature. If children do not have the opportunity to play with their peers because they cannot move well, it poses a problem to their self-esteem, which can then lead to further problems.

Another concern of researchers is that children with movement difficulties are less active than their peers (Bouffard et al., 1996). Bouffard et al., (1996) conducted a study during recess time in a schoolyard. They looked at 52 subjects to determine whether children with movement difficulties did in fact participate less in more-vigorous activities, played less often with large playground equipment, and spent less time in positive social interactions with others of their own gender. Although this study did support an activity deficit hypothesis in children with DCD, Bouffard et al. (1994) cannot be certain that $\mathrm{DCD}$ caused the activity deficit, because possibly the reverse occurred, where decreased activity levels may have put children at an increased risk of developing DCD.

Marchiori et al. (1987) indicated that a decrease in the amount of time spent on physical activity leads to a lack of practice time to perfect the movement skills. This results in a more defined performance difference from one's peers. As well, if this decrease in physical activity is allowed to continue, physical fitness levels will severely decrease, leading to health risks and possibly, social isolation. 


\section{KINEMATIC STUDIES}

Two kinematic studies (Hsu et al., 1989; Marchiori et al., 1987) have looked at children's movement during a particular task. Both studies included at least one child with movement difficulties. Marchiori et al. (1987) filmed subjects using a Photo Sonics 16 $\mathrm{mm}$ IPL camera at 100 frames per second and with another camera at 150 frames per second due to the rate at which one of the subjects performed. The cameras were placed perpendicular to the plane of motion 14 feet away. Hsu et al. (1989) used a Photosonics IP $16 \mathrm{~mm}$ high-speed camera set at 100 frames per second mounted perpendicular to the treadmill 12 feet away.

Marchiori et al. (1987) had subjects perform a stationary hockey slap shot as hard as they could while the linear velocity of the puck and angular displacement and angular velocity of the hockey stick were measured. Baseline data were taken for both groups (classified as "physically awkward" and "normal"). The second part of the study had the physically awkward children practice 40 trials for 5 days per week over a 6-week period. Data was collected every 2 weeks at 400,800 , and 1200 trials. Results indicated that the boys classified as "normal" achieved some consistency in their movement patterns. One subject, who had been enrolled in a hockey program for one year, had developed an even more consistent movement pattern. However, even after 1200 practice trials, the physically awkward boys still exhibited extreme variability.

The problem with this study was that the practice sessions were not under the supervision of the researchers. Therefore discrepancy between the subjects' practice 
sessions could exist. As well, only two boys formed each category, possibly, not providing a large sample size.

The second study was done by Hsu et al. (1989) to investigate associated movements in children. The researchers hypothesized that older children would show less associated movements than younger children. They added a motorically awkward subject the same age as the older children to serve as an intermediate between the two. A second objective of this experiment was to investigate the usefulness of angle/angle and phase-plane plot diagrams to see if they were relevant to associated movements. Children were instructed to walk both normally and in a heel walk with toes pointed in the air on a treadmill at a speed set by the researcher. No instruction was given regarding upper body limb movements. Joint angles were studied at the ankle, shoulder, and elbow. Results supported the hypothesis that the motorically awkward subject ranked between the younger and older subjects on most of the variables studied. The authors believed that cinematography was useful in obtaining information on children who are physically awkward and suggest that standardization should occur for angle/angle and phase-plane plot diagrams because of their usefulness in providing a visual pattern of the movement (Hsu et al., 1989). This particular study raised questions regarding the way children with DCD normally walk, as the associated movement patterns were an intermediate to an older and younger normal subject.

Surprisingly, only these two kinematic studies have been conducted with the inclusion of at least one child with a motor difficulty. Included in the study by Hsu et al. (1989) were some advantages of using cinematography as a means of data collection: it 
is noninvasive and requires no electrodes, wires, or any other apparatus other than paper joint markers attached to the subjects; multiple measurements involving more than one limb can be made in a single observation; and finally, the data can be digitized and stored for future analysis. Therefore, a biomechanical analysis of a movement skill, such as walking, would be useful when studying children with DCD.

\section{GAIT}

Walking is one of the most common activities that people do each day of their lives. Walking is a "method of locomotion involving the use of the two legs, alternately, to provide both support and propulsion" (Whittle, 1991, pg. 48). Learned early in the developmental stage, this method of locomotion takes us through later life. Unless born with a disability or an injury has occurred, it is one of the most overlooked movement skills. Upright locomotion is one of the key features that separates humans from other animals. Included in this review are historical perspectives and current technologies involved in gait analysis, the gait cycle, and gait patterns of children.

\section{Historical Perspective and Current Technologies involved in Gait Analysis}

Studying the human walking pattern from a truly scientific perspective began in the early $17^{\text {th }}$ century. A study by Borelli "measured the center of gravity of the body and described how balance is maintained in walking by constant forward movement of the supporting area provided by the feet" (Whittle, 1991, pg. 49). In 1936, the Weber 
brothers gave the first description of the gait cycle using only a watch with a second hand and a level piece of ground with a known distance (Deluzio, 1997).

However, it has only been in the latter part of the $20^{\text {th }}$ century that gait analysis has received the attention of many researchers. Due to the introduction of computers, motion tracking systems, and instrumentation, data collection and analysis have become relatively easier and quicker (Deluzio, 1997). Many more gait characteristics can be calculated using only what researchers now call "simple technology."

Gait can be studied using many different tools, the most basic being the naked eye. This method, however, is extremely dependent on the observer's ability to detect small changes and is limited to kinematic observations. Videotape techniques impacted gait analysis immensely by enabling the production of a permanent record of the movement and being able to detect high-speed events (Whittle, 1991). Biden, O'Connor, and Collins (1990) suggest that "limb segment orientations and joint angles are usually measured with cine-film, by video-based systems, or by goniometry (which measures limb orientations directly)" (pg. 294). Current technology has evolved to the use of video cameras that are directly linked to a computer. This method makes it quite easy to analyze any movement pattern because the software digitizes the marker patterns.

A major concern of researchers using technological equipment is the large amount of data produced. According to Deluzio (1997) "the major difficulties are how to handle the large quantity of measured variables, their time varying and highly correlated structure, and the non-linear nature of human gait" (pg. 16). Deluzio (1997) suggests there are two different methods of analyzing the waveform data. Parameter-based 
analysis is characterized by extracting values from the waveform such as the peak values or magnitudes at key cycle events, while waveform-based analysis characterizes the entire waveform. Both methods are different, yet the main goal is the reduction of the waveform data.

Both kinematic and kinetic information are important to the understanding of movement. Forces produced by the body are an important factor when studying gait patterns. Force plates are based on the assumption of Newton's Law of Reaction, that proposes for every action, there is an equal and opposite reaction (Hall, 1995). Force plates (or force platforms) "can be used to define the magnitude and direction of the resultant ground reaction force (GRF) applied by the ground to the foot" (Biden et al., 1990, pg. 298). Force plates measure the amount of force produced by the body on the floor in the horizontal, vertical, and lateral axis. Studies looking at gait patterns typically include information on vertical force, fore/aft shear, medial/lateral shear, torque, and information regarding the center of pressure (Sutherland et al., 1998).

The final medium for gait analysis involves electromyography (EMG). EMG consists of recording electrical signals associated with muscle activity (Biden et al., 1990). There are two types of electrodes that are used to record EMG; surface electrodes and intramuscular needle electrodes. Surface electrodes are more convenient and cause little pain, but they can pick up signals from neighboring muscles. 


\section{Gait Cycle}

Walking is clearly a repetitive, cyclical movement that has easily identifiable characteristics. Figure 2.1 illustrates the typical gait cycle of a normal 7-year-old child. A gait cycle is referred to as the "movements and events that occur between successive footsteps of the same foot" (Sutherland et al., 1988, pg. 16). Normally, researchers use heel strike to denote the beginning of a gait cycle as it is a clearly identifiable movement. There are two stages of gait: swing phase and stance phase. The stance phase comprises approximately $60 \%$ of the gait cycle and includes heel contact, foot flat, mid stance, heel off, and toe off. The swing phase includes the pendulum-like movement of the leg when not in contact with the ground and comprises approximately $40 \%$ of the gait cycle (Whittle, 1991). In order to compare subjects, time in the gait cycle is usually expressed as a percentage of the total cycle.

"Although some variability is present in normal gait, particularly in the use of the muscles, there is a clearly identifiable 'normal pattern' of walking, and a 'normal range' can be defined for most of the measurable parameters" (Whittle, 1991, pg. 91). Abnormalities in gait patterns are usually the result of a pathology. Walking is the result of a complex interaction between the brain, spinal cord, nerves, muscles, joints, and skeleton (Whittle, 1991). A disturbance to any of the systems can cause an abnormality in the individuals' gait pattern. Walking is clearly one of the most important movement patterns humans possess. "Understanding human locomotion is linked to understanding man" (Deluzio, 1997, pg. 10). 


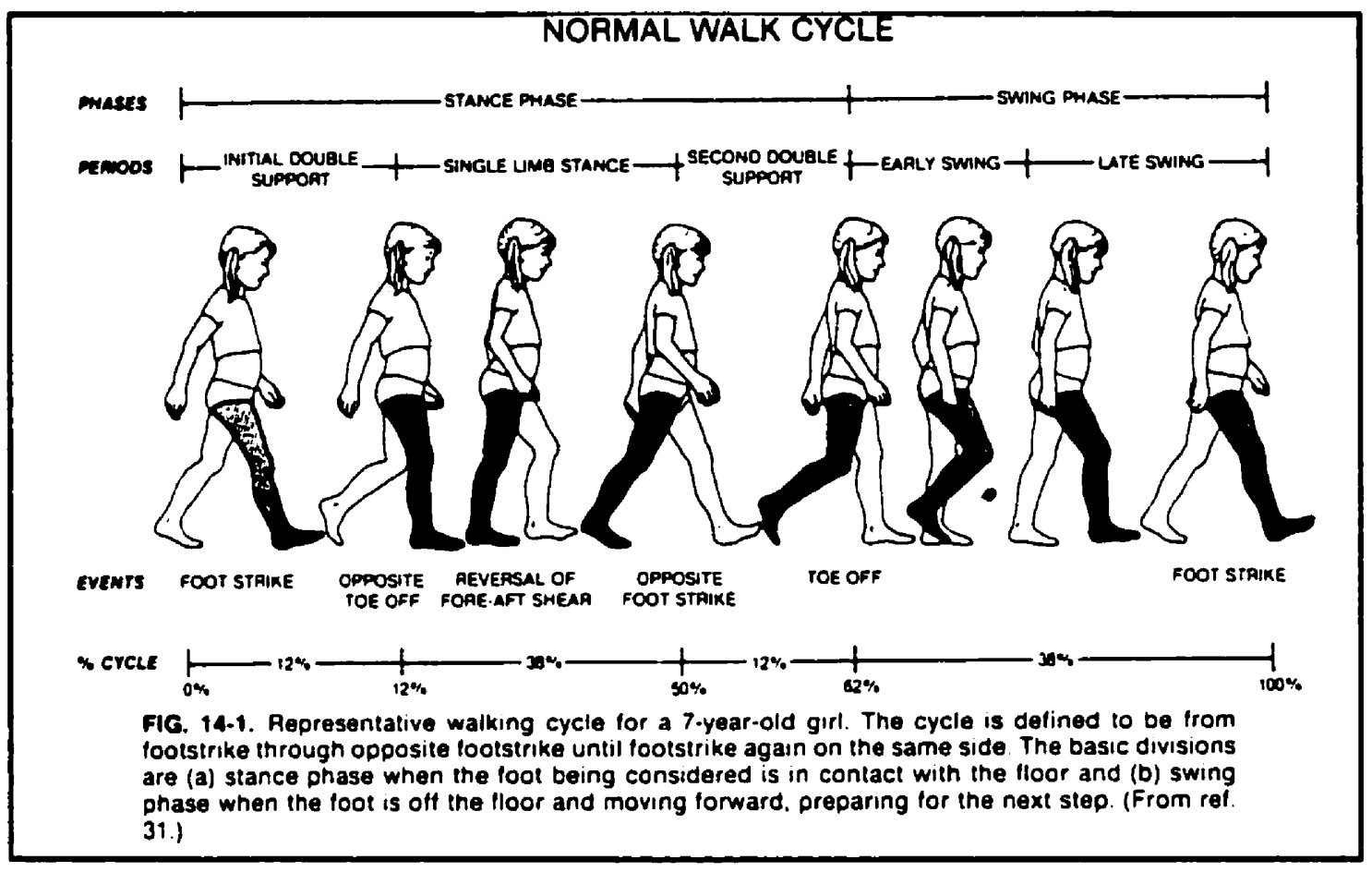

Figure 2.1 - Seven-Year-Old Gait (Sutherland et al., 1988, page 17)

\section{Gait Patterns of Children}

Gait patterns of children with cerebral palsy, the elderly, and individuals with below-knee amputations have been studied extensively and researchers have shown that gait in those individuals deviates from the normal pattern (Hall, 1995). Childhood presents an opportune time to study the development of gait patterns. Children are constantly changing and those changes can be seen over a span of time. Children's gait patterns differ from adult patterns by:

a wider base of support, stride length and velocity are lower and the cadence is higher, children have no heel strike, initial contact is made by the flat foot, there is very little stance phase knee flexion, the whole leg is externally rotated during the swing phase, and there is an absence of reciprocal arm swinging (Whittle, 1991, pg. 85-86). 
Most of these observable movements have matured by the age of four (Whittle, 1991), while Sutherland et al. (1988) indicated that gait patterns have matured by the age of three. However, cadence, stride length, and velocity continue to change with growth, until 15 years of age (Whittle, 1991), as these variables have a high correlation with lever lengths.

Steinwender, Saraph, Scheiber, Zwick, Uitz, and Hackl (2000) investigated the repeatability of gait analysis data in normal and spastic children. Lower repeatability in the gait data was found for spastic children, while the normal children displayed less variability. The repeatability of kinetics was better than those of kinematics for spastic children. Unintentional marker placement errors lowered the between-day repeatability of the gait data for both groups of children.

Sutherland et al. (1988) attempted to study gait patterns across the childhood age span. The purpose of their study was to find normative values of gait patterns in children. The study conducted at the Motion Analysis Laboratory at the Children's Hospital and Health Center in San Diego included 415 normal children (210 male and 205 female) ranging from ages 1 to 7 , in 10 different age groups. The study included kinematic, kinetic, and electromyographic variables. Four cameras (two placed sagittally of the walkway, one in front of the walkway, and one underneath the walkway in the force plate) were used in the data collection. Children were unaware of the force plate in the walkway so they would not alter their gait pattern. Electromyographic data were collected separately from the video and force plate data. Children included in the study were considered normal, and strict criteria were set to ensure the normality. The children 
in the study were a product of a full-term pregnancy, born at or after 38 weeks of gestation, walked independently by 14 months, had no orthopaedic problems or treatments, displayed normal growth and development as judged by both parents and family physicians, and had to have experienced no major medical problems or hospitalizations (Sutherland et al., 1988, pg. 30). The establishment of normative values of gait characteristics is extremely important and useful for researchers and clinicians to facilitate the amount of abnormality other children may have.

In the past, gait analysis has been studied extensively and has proved to be effective in describing walking patterns. Researchers have continued to study gait of different groups of individuals. Although the technology has advanced, it has not been perfected. The real difficulties of gait analysis lie in the need for clinical gait applications.

\section{WILSON'S ONE-DIMENSIONAL MEASURE OF NORMALITY}

Gait analysis can produce much data. Current researchers are uncertain of how to best extract meaningful data. As Deluzio (1997) writes:

In order to quantify gait, numerical data have been sometimes considered the same as knowledge. The best that may occur under such a paradigm is that within the accumulated data, numbers are found that increase understanding and knowledge. It is more likely, and gait analysis has been criticized for this (Brand, 1992), that the researchers become overwhelmed with data without knowing how to extract meaningful information. This situation is present in gait analysis today (pg. 9).

Gait patterns are often studied in pathological populations, or in such cases as pre and post surgeries to identify changes due to the intervention program. Most often, 
researchers need to be able to quantify what abnormal gait patterns are as compared to normal deviations. Wilson's (1998) purpose was to describe the variation from the mean seen in a normal population (Sutherland et al., 1988), as well as variations from the mean which are indications of pathological gait. Wilson's (1998) goal was to seek measures which spread the data out and which produce clear distinctions between normal and abnormal conditions.

Wilson (1998) chose the sagittal hip, knee, and ankle flexion/extension variability, although the technique is applicable to other combinations of curves. The analysis included 174 observations of children aged 3 to 7 , as work done by Sutherland et al. (1988) suggested that gait patterns have matured by the age of 3 . The gait patterns were recorded for both the left $(n=174)$ and right $(n=173)$ sides and were combined to form one set of 348 observations. By pooling the left and right data, variances may be underestimated due to the pooling of "between child" variation and the "within child" variation. For each cycle, a set of $\mathrm{T}$ frames was chosen, corresponding to approximately evenly spaced time periods throughout the cycle. At each of the T times, angle displacement measures were recorded for sagittal hip, knee, and ankle flexion/extension.

As there is no obvious way to compare the set of angle displacement curves for a number of cycles with others of different frame numbers, the angle measurements from each recorded gait cycle were replaced by a set of Fourier coefficients, using the same number of coefficients for every cycle. A set of 12 Fourier coefficients ( 6 harmonics) was chosen to give an adequate approximation to the gait data. Each subject's angle displacement measurements were recentered to average out to zero for each joint. 
Therefore the observed displacements about the averages have been approximated in equation 2.1 by a 36-dimensional vector, $\underline{\Gamma}^{(i)}$, for each of the 3 joint angle rotations, hip

$$
\Gamma^{(i)}=\left(\chi_{h}^{(i)}, \chi_{k}^{(i)}, \chi_{a}^{(i)}\right) \quad i=1, \ldots 348
$$

flexion/extension $(h)$, knee flexion/extension $(k)$, and ankle dorsi flexion/extension $(a)$, as recorded from a typical cycle from one side of a child.

Each observation, $\Gamma^{(i)}$, is considered a random vector, centered about a population mean vector $\bar{\Gamma}$ (average of 348 observed $\underline{\Gamma}^{(i)}$ ). A sample covariance matrix, $\hat{\Sigma}_{r}$ (36 by 36) is calculated using equation 2.2. The $(k, l)$ entry of $\hat{\Sigma}_{r}$ describes the covariance of the $k$ th element of the $\underline{\Gamma}^{(i)}$ with the lth element. The diagonal entries in the covariance matrix are simply the sample variances of the corresponding components of the $\underline{\Gamma}^{(i)}$ vectors.

$$
\begin{gathered}
\hat{\Sigma}_{\mathrm{r}}=\frac{1}{347} \sum_{i=1}^{348}\left(\Gamma^{(i)}-\bar{\Gamma}\right)^{T}\left(\Gamma^{(i)}-\bar{\Gamma}\right) \\
(k, l) \text { entry of } \hat{\Sigma}_{\Gamma}: \frac{1}{347} \sum_{i=1}^{348}\left(\Gamma^{(i)}-\bar{\Gamma}\right)_{k}\left(\Gamma^{(i)}-\bar{\Gamma}\right)_{l}
\end{gathered}
$$


The total variation in the training set is calculated as 347 times the sum of the diagonal entries of $\hat{\Sigma}_{\Gamma}$. Note that the total variation can also be written as the sum of the squares of the lengths of the recentered $\underline{\Gamma}^{(i)}$

$$
\text { Total variation }=\sum_{i=1}^{348}\left\|\Gamma^{(i)}-\bar{\Gamma}\right\|^{2}
$$

The next step involved reducing the dimensionality of the problem without losing much information. Deluzio, Wyss, Zee, Costigan, and Sorbie (1997) used principal component analysis, a multivariate statistical procedure, as a data reduction technique. This type of analysis involves searching for directions in which the data shows the most variability. Principal components are linear combinations of the original variables and represent orthogonal directions in a multi-dimensional space of the variables. However, most principal components only capture a small amount of the total variation (Wilson, 1988). Wilson (1998) was able to explain $75 \%$ of the total variation in 348 trials using 11 interpretable functions while, Deluzio et al., (1997) explained the variation in 30 trials using 22 interpretable functions. It would have been more appropriate to develop a correlation matrix for the 22 variables to further reduce the dimensionality. As principal components are difficult to interpret and not of practical use or interest without interpretation, an alternative approach would be to find a set of interpretable functions which explain most of the variation explained by the principal components.

The first 4 principal components were calculated to explain $72.7 \%$ of the total variation, while the first 8 principal components were calculated to explain $85.7 \%$ of the 
total variation (Wilson, 1998). These numbers were used comparatively to the interpretable functions in hope of explaining at least $70 \%$ of the variation. A set of 11 interpretable functions was calculated to explain $74.8 \%$ of the variation in the 36 dimensional database. The 11 interpretable functions included: mean angular hip displacement, mean angular knee displacement, mean angular ankle displacement, mean angular hip velocity, mean angular knee velocity, mean angular ankle velocity, mean angular hip acceleration, mean angular knee acceleration, mean angular ankle acceleration, and two prime frequency numbers (with values of $\alpha_{1}$ and $\beta_{1}$ ) for mean angular knee and ankle displacement.

The numeric values of the interpretable functions formed an 11 by 36 matrix, $Q$. Each subjects $\underline{\Gamma}^{(i)}$ was approximated by a linear combination of the interpretable function:

$$
\underline{\Gamma}^{(i)} \doteq Q^{\top} \underline{B}^{(i)}
$$

Then standard techniques of linear algebra were used to calculate a squared distance measure, $D^{(i)}$, comparing each 11 -dimensional row vector, $\underline{\mathrm{B}}^{(i)}$, with the zero vector (equal to the average). The $D^{(i)}$ measure is the one-dimensional measure of normality. However, if the 11 -dimensional $\underline{B}^{(i)}$ was to follow a multivariate Normal distribution, then the measures, $D^{(i)}$, should follow a Hotelling's $T^{2}$ distribution. The observed values $N^{(i)}$ defined by equation 2.5 would be distributed as $\mathrm{F}_{\alpha, m-d+l}$, where $d=11$ is the dimension 
of the observations $\underline{B}^{(i)}$, and $m=348$ is the number of observations. The rescaled measures

$$
N^{(i)}=D^{(i)} \frac{(m-d+1)}{m d}
$$

of normality, $N^{(i)}$, should be distributed as $\mathrm{F}_{11,338}$. Wilson (1998) needed to find cut-off values in order to quantify abnormality. Non-parametric 95 percentile upper confidence bounds were calculated for the $95^{\text {th }}$ percentile, and the value 2.31 was chosen as the upper bound on normality: any subject having a measure greater than 2.31 should be declared as abnormal. Using the normative database, the observed $95^{\text {th }}$ percentile was chosen as the lower bound on normality: any subject scoring less than 1.73 should be declared normal. If the measure falls between 1.73 and 2.31 , the subject should be declared unusual and be further investigated. Refer to Figure 2.2 for a summary of the formulation of the Wilson Score.

The normative one-dimensional statistic was then tested using very young children and children up to 7 years of age who were bom prematurely. This type of classification was able to discriminate between normal and abnormal gait patterns. Very young children, just learning to walk, should indeed have very different gait patterns. Nearly $90 \%$ of these subjects were classified as unusual or abnormal. Similar results were shown with children who were born prematurely. 

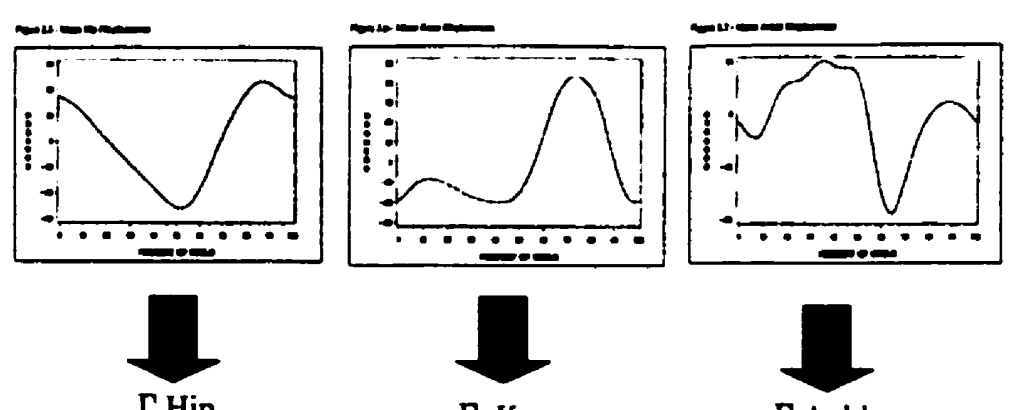

$\Gamma$ Knee

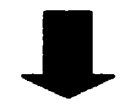

$\Gamma$ Ankle

12 Fourier Coefficients

12 Fourier Coefficients

12 Fourier Coefficients
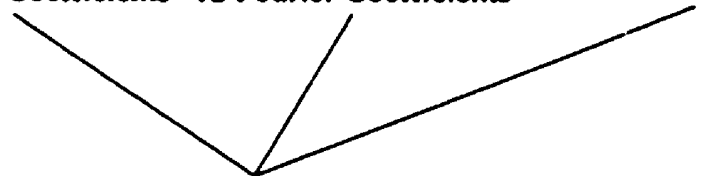

II dimensional approximation

II dimensional

score

Figure 2.2 - Summary of the Formulation of the Wilson Score

However, Wilson (1998) only used sagittal hip, knee, and ankle observations, which may be difficult for clinicians to ascertain, as they typically do not have the same type of equipment to record gait observations as researchers. Therefore, time/distance variables such as cadence, stride length, and percentage of toe off should be developed using similar methodology as Wilson (1998), as these measures are typically used and easy to record.

\section{SUMMARY}

Movement is essential for all individuals, especially children who are learning to move about in their environment. If children are not able to move well, their experiences will hinder future experiences, thus participating in a less-active lifestyle. Children with DCD 
have attracted the attention of researchers because of their negative experiences with movement and movement skill acquisition. Geuze and Borger (1993) reported that approximately $50 \%$ of children diagnosed with DCD early in life still show existing signs into adolescence. The signs of clumsiness are accompanied by increased social and emotional problems for children with DCD. Therefore, it is imperative to help children diagnosed with $D C D$ as early as possible so that they may find ways to enjoy physical activity.

Children with DCD should be initially screened by using a simple, but valid and reliable measurement to infer a problem with daily activity and academic achievement. This can be done using a criterion-referenced test by the teachers within a normal classroom environment. For the purpose of identifying a serious motor impairment, a normative-referenced test should be used. The Movement $A B C$ package (Wright and Sugden, 1992) provides a good and practical tool for researchers in attempting to identify children with DCD.

Two kinematic studies (Hsu et al., 1989; Marchiori et al., 1987) have been done in the past which have included at least one child showing signs of DCD. Both of these studies show that kinematic evaluation is beneficial to the researcher. More studies of this kind are needed to create a clearer picture of the children's movement patterns.

In normal human development, running, skipping, hopping, and galloping all emerge from a stable, independent walking pattern. Children with DCD are normally diagnosed if there is a motor impairment to either fine or gross motor skills. Therefore, 
an impairment to walking patterns maybe an underlying cause of the difficulties that children with DCD face.

Gait patterns in children have been widely studied in the past. The gait cycle has clearly identifiable patterns that can be studied and compared to other individuals. Childhood presents an opportune time to study the development of gait patterns, as children are constantly changing and changes can be seen over a large span of time. Sutherland et al. (1988) attempted to identify gait patterns across childhood. Kinematic, kinetic, and electromyographic variables were included in the normative database.

Current researchers are faced with the question of how to extract meaningful data from the large amounts of data that are produced from a gait analysis. Wilson (1998) investigated a one-dimensional measure of normality based on the sagittal hip, knee, and ankle curves. This type of classification discriminated between normal, abnormal, and unusual gait patterns of children classified as normal, children classified as normal under the age of three, and children up to the age of seven that were born prematurely. 


\section{CHAPTER 3}

\section{METHODOLOGY}

\section{INTRODUCTION}

The purpose of this study was to validate a statistical procedure used to discriminate abnormal gait patterns from normal gait patterns. The procedure has already been tested on children that have atypical walking patterns that are apparent to the naked eye. This statistical procedure would hopefully discriminate atypical walking patterns from a population that may not be so apparently abnormal using only naked eye observation. Children with DCD from grade one classrooms were selected to be included in this study. To ensure a proper diagnosis in accordance with the DSM-IV (1994) definition of $\mathrm{DCD}$, the Movement $\mathrm{ABC}$ was chosen because it has both normative and criterionreferenced sections. The normative-referenced section was used to remove any doubt of a serious motor impairment and the criterion-referenced section addressed problems of daily living. The Movement $A B C$ is one of the tests most often used by researchers (Hoare,1994; Piek and Coleman-Carman, 1995; Smyth and Mason, 1997; Wright and Sugden, $1996 \mathrm{a}, \mathrm{b}$ ). According to the MABC manual, a child scoring at the $15^{\text {th }}$ percentile indicates moderate $D C D$ while the $5^{\text {th }}$ percentile indicates severe $D C D$. Therefore, for the present study, any children scoring at or below the $15^{\text {th }}$ percentile on the MABC Checklist and Test (Henderson and Sugden, 1992) were included. The children who met the diagnostic criteria were then asked to participate in the study. 
Children with DCD were invited to the Gait Laboratory at the University of New Brunswick for motion analysis. A 3-camera Vicon system was used to capture the gait of children with DCD. The time/distance variables (percentage of the cycle at which opposite toe off occurs, percentage of the cycle at which opposite foot strike occurs, percentage of single stance, percentage of the cycle at which toe off occurs, and stride length, cycle time, cadence, and walking speed), sagittal hip flexion, sagittal knee flexion/extension, and sagittal ankle flexion/extension were all used for data analysis. Sagittal hip flexion, knee flexion/extension, and ankle flexion/extension were scored using Wilson's (1998) procedure. Furthermore, Wilson's (1998) methods were used to create a time/distance score (Fscore) using the data of the San Diego database (Sutherland et al., 1988). Children with DCD were given a Fscore, to determine whether they had normal, abnormal, or unusual gait patterns.

\section{SUBJECTS}

Children in grade one (six and seven years old) were subjects for the present study. Children at this school level have had time to adjust to normal school days, however they are still young enough to be receptive to an intervention strategy. Sutherland et al. (1988) indicated that walking patterns are typically mastered between the ages of three and four, with the exception of the time/distance variables. The time/distance variables are measures of lever lengths and therefore do not mature until adulthood. However, time/distance variables are very common in gait research and are typically easy to measure. 
An important element in gait analysis is the availability of normative data against which to compare the study data. The Institute of Biomedical Engineering at the University of New Brunswick has access to one of the largest available normative databases for children's gait. The study that produced this data was conducted at the San Diego Children's Hospital in San Diego, California (Sutherland et al., 1988). This database includes kinetic, kinematic, and electromyographic data as well as anthropometric and developmental milestones for children ages one to seven years old (Sutherland et al., 1988). The kinematic information for the subjects included in the San Diego database were compared to the data for children with DCD.

\section{DATA COLLECTION}

\section{Identification of DCD}

The testing instruments used in this study were the Movement ABC Test and Checklist (Henderson and Sugden, 1992). All protocols in the Test manual were adhered to closely.

The MABC Checklist (Henderson and Sugden, 1992) was used as a screening tool to be filled out by the child's homeroom teacher. This instrument looks at both the child and the environment in which the task is being performed. The MABC Checklist is divided into five sections: child stationary/environment stable, child moving/environment stable, child stationary/environment changing, child moving/environment changing, and behavioral problems related to motor difficulties. The first 4 sections are answered on a scale of 0 to 3, 0 indicating very well and 3 indicating not close. The behavioral section 
of the test is scored on a scale of 0 to 2, 0 indicating rarely and 2 indicating often. Cut-off scores in the manual were used to determine if the MABC Test was necessary.

The MABC Test (Henderson and Sugden, 1992) was used as a normative-

referenced assessment tool. It is subdivided into three subsections: manual dexterity, ball skills, and static and dynamic balance. The MABC Test is organized for different age bands. For the purpose of this study Age Band I ( 4 to 6 years old) and Age Band II ( 7 to 8 years old) were used. Each child was permitted one attempt at each item. However, if a failure occurred, further attempts were permitted, in accordance with the MABC Manual (Henderson and Sugden, 1992).

A health/activities questionnaire was formulated using the same questionnaire as in the San Diego database. The questionnaire asked questions regarding the medical history of the child. Also asked, was questions regarding the child's interests, hobbies, and current organized activities. The questionnaire was sent home with the potential subjects with the parental consent forms. Refer to Appendix A for the health/activities questionnaire.

\section{Gait}

Gait was analyzed using a camera-based system to measure the displacement of reference markers located on the child's skin. A Vicon 140 motion analysis system (Oxford Metrics Ltd) comprised of 3 infrared cameras was used. The cameras were positioned in a task-specific configuration about a calibrated work space area. The first camera was placed approximately 3 metres away from and perpendicular to the center of the 
walkway. The remaining two cameras were placed approximately 60 degrees on either side of the first camera. Refer to Appendix B for a diagram of the Gait Laboratory. The cameras recorded the gait cycle at a sample frequency of $60 \mathrm{~Hz}$, which is comparable to the sampling frequency of $50 \mathrm{~Hz}$ used in the San Diego database (Sutherland et al., 1988).

\section{PROCEDURES}

\section{Identification of DCD}

Standard procedures were used to obtain necessary approval for this study from the Director of School District 18 (refer to Appendix C) and the principals of each school involved. As well, ethical approval was obtained from the Faculty of Kinesiology at UNB (refer to Appendix D). Parental consent was obtained before any contact was made with the children. A letter stating the nature of the study as well as all procedures accompanied the parental consent form. Refer to Appendix E for the letter and consent forms. A health/activities questionnaire was sent with the consent form for the parent or guardian to complete (Appendix A). The MABC Checklist was first administered by the classroom teacher to children whom the teacher thought had difficulties with fine and/or gross motor movements. The Checklist was left with the teacher for one week or until completed.

Following the calculation of the results of the Checklist, students were notified for further testing. The MABC Test was then administered in a one-to-one testing situation at the child's school. The MABC Test took approximately 30 minutes per child to administer. 


\section{Gait}

Children identified as having DCD were invited to the Gait Laboratory at UNB. The subjects were asked to wear shorts during the testing session. Lightweight reflective markers were placed on the left and right lower extremities in accordance with the San Diego marker set (Sutherland et al., 1988). The position of the 17 markers on the subjects' skin were as follows: anterior superior iliac spine (ASIS), greater trochanter, mid-thigh, femoral condyle, mid-calf, malleolus, heel, $2 / 3$ metatarsal, and sacrum. The mid-thigh, mid-calf, and sacral markers were mounted on sticks and secured with velcro straps to reduce co-linearity and position measurement. The markers used to calculate the angles for the measurements in this thesis include the greater trochanter, femoral condyle, malleolus, heel, $2 / 3$ metatarsal, and sacrum.

The 3-camera Vicon motion capture system recorded the left and right sides of the participants as they walked back and forth on a 13-foot walkway at a self-selected pace. The cameras were positioned so that the subjects were allowed to reach normal walking speed before they entered the data collection area. The data collection area allowed 1 to 3 complete gait cycles to be captured, depending on the child's age and height. The subject was asked to walk until 16 gait cycles were captured ( 8 cycles per side).

In order to choose which trial to use for data analysis, the time/distance variables were averaged over the trials captured. The individual trial which best represented the averaged trials was used for data analysis. This method is similar to the original San Diego database protocol (Sutherland et al., 1988). 


\section{CALCULATION OF THE FSCORE}

One of the objectives of this thesis was to develop a one-dimensional measure of normality using time/distance variables. The data for 139 normal children aged 3, 3.5, 4, 5,6 , and 7 were combined to form the basis for the training set. Gait patterns of children under the age of three have more or less immature patterns and therefore were excluded from the training set. As well, the data from children with missing data or a suspected recording error were excluded. For children who had data recorded for more than one age, the data for the greatest age were included. Please refer to Appendix $F$ for a complete list of children who were excluded.

The time/distance variable information contained the percentage of opposite toe off, percentage of opposite foot strike, percentage of single stance, percentage of toe off, step length $(\mathrm{cm})$, stride length $(\mathrm{cm})$, cycle time $(\mathrm{sec})$, cadence (steps $/ \mathrm{min})$, walking speed $(\mathrm{cm} / \mathrm{sec}, \mathrm{m} / \mathrm{min})$, and the presence or absence of a right heel strike, left heel strike, or reciprocal arm swing (yes/no). However, certain measured variables seemed redundant as they were merely measures of other variables. Examples include cadence, which is a measure of step length and walking speed; cycle time, which is a measure of stride length and walking speed; and stride length, which can be calculated by virtually doubling step length. As well, there were measures of walking speed recorded in both $\mathrm{cm} / \mathrm{sec}$ and $\mathrm{m} / \mathrm{sec}$.

Therefore cadence, cycle time, stride length, and walking speed $(\mathrm{cm} / \mathrm{sec})$ were omitted from the training set. Other measures that were omitted are seen in Table 3.1. The data included for the presence or absence of a right heel strike, left heel strike, and 
reciprocal arm swing was recorded as a "yes/no" answer. Since almost all responses were "yes," these variables did not separate abnormal from normal data, so these variables were dropped from the training set.

Table 3.1

Presence or Absence of a Right Heel Strike, Left Heel Strike, and Reciprocal Arm Swing

\begin{tabular}{lcccc}
\hline & Yes & No & Total & Missing Data \\
\hline Right Heel Strike & 150 & 0 & 150 & 0 \\
Left Heel Strike & 146 & 3 & 149 & 1 \\
Reciprocal Arm Swing & 150 & 0 & 150 & 0 \\
\hline
\end{tabular}

Finally, percentage of opposite foot strike was omitted because the data were discrete, as described in Table 3.2. The data recorded had all points between 49 and $52 \%$ of the gait cycle, therefore discriminating between abnormal and normal patterns was not possible. The major concern was that the total spread of the data was comparable to the noise level (or error level) in the measurement and that the distance measures calculated in this thesis were more appropriate using continuous data.

The variables that remained were percentage of opposite toe off, percentage of single stance, percentage of toe off, step length, and walking speed. However, as Wilson (1998) indicated, "walking velocity (speed) is highly correlated with age, as the older a child is, the faster he or she walks" (pg.7), therefore walking velocity has been excluded from the training set. As well, because of this statement, step length was changed to a 
measure of percentage of the gait cycle rather than distance traveled. The measurement originally was recorded in centimetres, however, due to the correlation to age, step length was divided by stride length, in order to record it as a percentage of the gait cycle.

Table 3.2

Percentage of Opposite Foot Strike

\begin{tabular}{cccc}
\hline Left Side (\%) & Count & Right Side (\%) & Count \\
\hline 49 & 41 & 49 & 25 \\
50 & 64 & 50 & 66 \\
51 & 36 & 51 & 51 \\
52 & 9 & 52 & 8 \\
$\mathrm{n}$ & 150 & $\mathrm{n}$ & 150 \\
\hline
\end{tabular}

Since the remaining data were recorded for both left and right sides, they were pooled together to form a larger sample size. In accordance with Wilson (1998), the sample size was inflated by treating left and right sides as one large sample $(n=278)$. Refer to Appendix $G$ for the correlation matrix for the left side data $(n=139)$, the correlation matrix for the right side data $(n=139)$, and the correlation matrix for the combined left and right sides $(n=278)$. For entries with strong correlations, the patterns were quite obvious, as shown in Figure 3.1, which depicts the correlation between percentage of opposite toe off and percentage of single stance. 


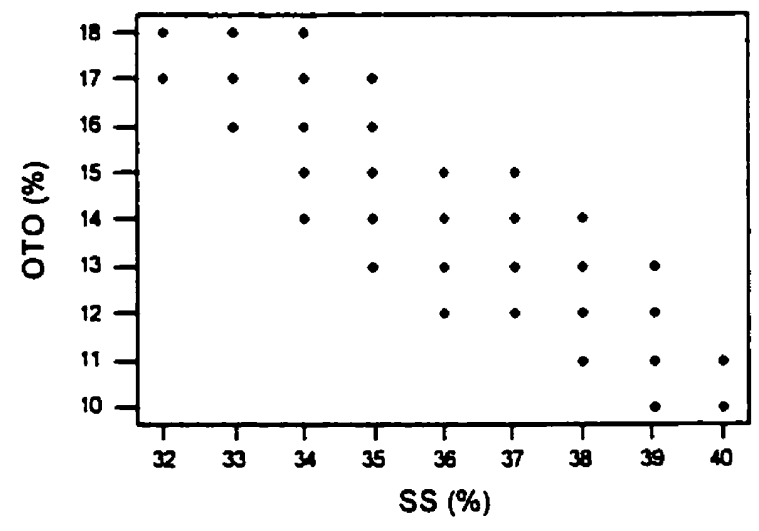

Figure 3.1 - Correlation between Percentage of Opposite Toe Off (OTO \%) and Percentage of Single Stance (SS \%) (Correlation $=-0.861)$

Hotelling's $T^{2}$ statistic was used by Wilson (1998) to combine children's scores into a single number while allowing for the known interaction pattern. For example, subject 8005 had values where opposite toe off occurs at $12 \%$ of the gait cycle (OTO = 12), $50 \%$ of the gait cycle is in single stance ( $S S=50$ ), toe off occurs at $61 \%$ in the gait cycle (TO $=61$ ), and has a step length of $49.5 \mathrm{~cm}(\mathrm{SL}=49.5)$ recorded for the left side. If only the information on opposite toe off was used to determine whether subject 8005 values fell within the "normal bounds," the usual t-test calculates:

$$
\text { Score }=\frac{\text { Observed Opposite Toe Off }- \text { Average Opposite Toe Off }}{\text { Standard Deviation }}=\frac{12-13.97}{1.65}
$$


A Score of -1.15 was calculated, which is inside the $95 \%$ prediction bounds for $t_{2 \pi}$. Subject 8005 would not be considered unusual based on opposite toe off $(\%)$. Wilson (1998, pg. 36) explained that $t_{27}$ was not really appropriate in this situation, though $t_{27}$ was a good apfroximation. Wilson (1998) suggested using non-parametric tolerance bounds rather than the values of a t statistic. These more complicated bounds allowed for the fact that the $\mathbf{2 7 8}$ observed gait cycles obtained from the San Diego database was merely a sample from all possible gait cycles which could have been observed on all normal children. In particular, the sample estimates of average opposite toe off (OTO = 13.97) and its standard deviation $(\mathrm{SD}=1.65)$ were surely different from the true population values. The algorithm for obtaining tolerance bounds was described in Wilson (1998) and calculated values were compared to statistical tables. That is, given sample size and confidence level, Wilson's (1998) algorithm was used to find the appropriate bound.

Instead of recalculating the above score (Equation 3.1) three more times, one would like to use a score that combines all four measures, considering the correlations with each other. As indicated above, the first step for calculating the one-dimensional measure of normality (Score1) included subtracting mean values from each individual value for each variable. Table 3.3 indicates the mean and standard deviation values for the four variables used to derive a one-dimensional measure of normal gait. 
Table 3.3

Mean and Standard Deviation Values of Opposite Toe Off (\%). Single Stance (\%), Toe Off (\%), and Step Length (\%)

\begin{tabular}{lccc}
\hline \multicolumn{1}{c}{ Variable } & $\mathrm{n}$ & Mean & Standard Deviation \\
\hline Opposite Toe Off (\%) & 278 & 13.97 & 1.65 \\
Single Stance (\%) & 278 & 36.22 & 1.63 \\
Toe Off (\%) & 278 & 64.00 & 1.68 \\
Step Length (\%) & 278 & 50.01 & 1.24 \\
\hline
\end{tabular}

In the example using subject 8005 , values were as follows:

$\begin{array}{ll}\text { OTO } 12.0-13.97 & =-1.97 \\ \text { SS } & 50.0-36.22=13.78 \\ \text { TO } 61.0-64.00=-3.00 \\ \text { SL } 49.5-50.01=-0.51\end{array}$

The values were then multiplied using equation 3.2 , where ${ }^{\top}$ represents the transpose (from a row vector to a column vector) and the 4 by 4 matrix $\operatorname{Cov}^{-1}$, calculated by pooling the data set of 278 gait cycles, is given in Appendix H. Subject 8005 had a Scorel of 3.51 .

$$
\text { Score } 1=[-1.97,13.78,-3.00,-0.51] \operatorname{Cov}^{-1}[-1.97,13.78,-3.00,-0.51]^{\mathrm{T}}
$$

Again, following Wilson's (1998) model, if the 4-dimensional time/distance vectors were to follow a multivariate Normal distribution, then Scorel measures should follow a 
Hotelling's $\mathrm{T}^{2}$ distribution. Using equation 3.3, those rescaled measures, Fscore, would be distributed as $\mathrm{F}_{d, m \cdot d+1}$, where $d$ is the dimension of the observations (in this case, $d=$ 4), and $m$ is the number of observations (in this case, $m=278$ ) (Wilson, 1998).

$$
\text { Fscore }=\operatorname{Scorel} \frac{(m-d+1)}{m^{*} d}
$$

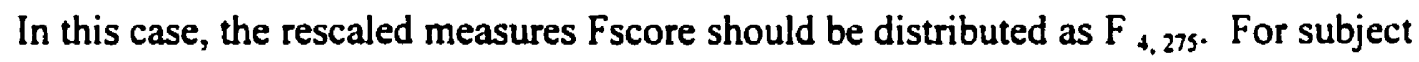
8005, the Scorel was calculated to be 3.51 and Fscore was calculated to be 0.87 . An Fscore value of 0 would indicate that the subject's time/distance variables perfectly matched the four averages listed in Table 3.3. Given the natural variability of the population, an Fscore of 0 would in fact be most unusual. The expected value (average) of any F statistic is 1 . So, typically, children would have had values of Fscore near 1. Values of Fscore considerably greater than 1 indicated abnormal gait.

Similar to Wilson (1998), the rescaled sets of measures, Fscore, were then sorted in ascending order. The values were plotted against the values of the appropriate $F$ distribution. If the points on the graph formed a straight line with a slope of about one, then the measures were distributed like the corresponding $\mathrm{F}$ distribution. This type of plot is called a Q-Q plot. Figure 3.2 illustrates a Q-Q plot of the measures, Fscore versus the quantiles of an $F_{4,275}$, which measures almost a straight line, resulting in a confident $F$ distribution. Interestingly, the observed $95^{\text {th }}$ percentile of the quantiles of $F_{+2.27}$ gave a value of 2.4 , whereas the observed $95^{\text {th }}$ percentile of 278 normative observations gave a 
value of 2.26 . It should be noted that the observed $95^{\text {th }}$ percentile of 278 normative observations included more observations than the observed $95^{\text {th }}$ percentile of the quantiles of $F_{4.275 .}$

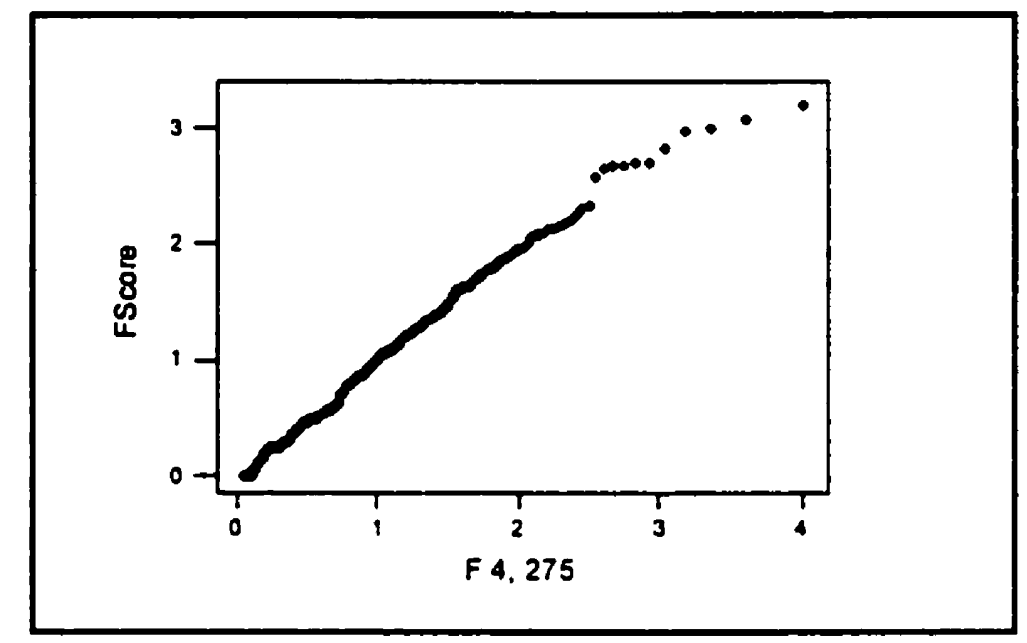

Figure 3.2 - Q-Q Plot: Observed Quantiles of $F$ versus the Quantiles of $F$ 4,275.

Finally, in order to describe the gait patterns, cut-off values were determined to quantify the patterns as normal, abnormal, or unusual. As described earlier, Wilson (1998) explained that non-parametric cut-off points need to be at least $95 \%$ confident that the cut-off is at least as high as the true population $95^{\text {th }}$ percentile of normal children and should be declared as the upper cut-off value of normality. Wilson (1998) chose to break down the data into left and right sides to ensure that the subject being classified as abnormal was truly abnormal. Wison (1998) chose to use the highest number between left, right, and both sides scores, to ensure that the children being described as abnormal, were truly different from those being described as normal. In that case, the largest 
number, 2.31 , was calculated from the right side indicating that the least amount of children were being classified as abnormal. To calculate the lower bound of normality, Wilson (1998) used the observed $95^{\text {th }}$ percentile from the normative database. Again, the left and right sides were calculated separately to ensure that all of the subjects being classified as normal were in fact normal. The left side gave a value of 1.73 which was used as the lower bound, declaring all subjects scoring below 1.73 as normal. A score between 1.73 and 2.31 , called "unusual" by Wilson, prompts researchers/clinicians to further investigate the gait pattern abnormalities. Wilson's (1998) technique classified 7 $\%$ of children in the unusual category.

For the purpose of this thesis, the combined left and right Fscores were used to calculate the bounds of normality. The non-parametric $95^{\text {th }}$ percentile gave an Fscore cutoff value of 2.69. In accordance with Wilson (1998), the non-parametric cut-off point should be used as an upper confidence bound, declaring that subjects whose scores were above 2.69 should be considered abnormal. The observed $95^{\text {th }}$ percentile, lower bound, was calculated to be 2.26 . In this case, children scoring lower than 2.26 should be declared normal. The area between the two points can be considered an unusual situation.

The cut-off values were calculated from data that should be behaving like $F_{4,275}$ as seen in Figure 4.2, the Q-Q plot. The $95^{\text {th }}$ percentile of $F_{4,275}$ gave a value of 2.4 , which falls between the two cut-off values. Therefore confidence was reassured as the $95^{\text {th }}$ percentile of $F_{4,275}$ fell between the two cut-off points. Table 3.4 indicates the classification results of the San Diego database results for both the Wilson score, and the 
Fscore. The Fscore was able to classify more children as abnormal, while the Wilson score classified more children in the unusual category. Refer to Figure 3.3 for a summary of the formulation of the Fscore.

Table 3.4

Classification Results of the San Diego Database (three to seven years old) for the Wilson score and the Fscore

\begin{tabular}{lcccc}
\hline & Wilson $^{\mathrm{a}}$ & \multicolumn{3}{c}{ Fscore $^{\mathrm{b}}$} \\
\hline Normal & 319 & $91.4 \%$ & 264 & $94.9 \%$ \\
Unusual & 25 & $7.2 \%$ & 6 & $2.2 \%$ \\
Abnormal & 5 & $1.4 \%$ & 8 & $2.9 \%$ \\
\hline
\end{tabular}

Note. $n=349$ children. ${ }^{\mathrm{b}} \mathrm{n}=278$ children. 


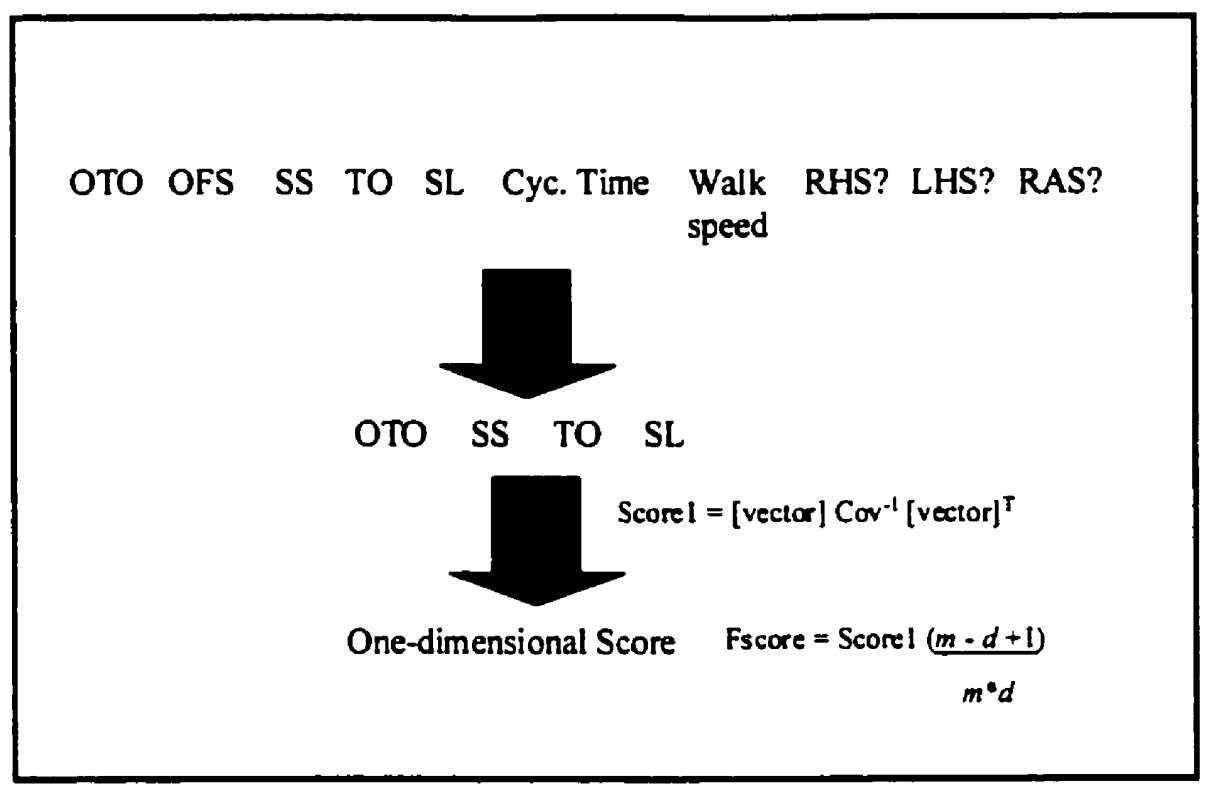

Figure 3.3 - Summary of the Formulation of the Fscore.

\section{DATA ANALYSIS}

The Vicon motion analysis system produced comma delimited files (.csv) that were processed using a program called RunAGait $\mathcal{O}$ Angle Calculation, Version 2.1.

RunAGait 0 was able to calculate all of the linear measurements using the San Diego database methods (time/distance measurements and angle curves). The appropriate variables were transferred into Minitab 12.1 (Minitab Inc., 1998) for the analysis of the Wilson score and the new score developed in this research. For the curves involved in the Wilson score, the angle curves were overwritten and filtered with the Fourier Coefficients. Wilson (1998) suggested looking at the 11 -dimensional $\underline{B}^{(i)}$ vector (interpretable functions) that is produced in the calculation of the score for the abnormalities. "The standardized vector describes 'standard deviations from the mean,' so that values above 2.0 or below -2.0 indicate unusual pattems" (Wilson, 1998, pg. 76). 
The statistical analyses in this study were performed using Minitab 12.1 Released for Windows ${ }^{\oplus}$ (Minitab Inc., 1998).

Analysis for Hypothesis 1: Using the one-dimensional measure of normal gait for the sagittal hip, knee, and ankle angular displacement developed by Wilson (1998), children with DCD were classified as normal, abnormal, or unusual. If most children with DCD were to be classified by the Wilson score as abnormal, as expected, discriminant analysis may be appropriate to determine whether children with DCD differ from the normal in a systematic way.

Analysis for Hypothesis 2: A covariance matrix was calculated from the time/distance variables using the original data from the San Diego database to identify which variables were predictable from others and which values seem to be independent. A restricted set of time/distance variables, not easily predicted from each other, were then used in subsequent analysis. Mean (Hypothesis 2(a)) and covariance structures (Hypothesis 2(b)) were then compared for the normative and children with DCD data. If the mean for the two groups was significantly different, then discriminant analysis may be appropriate to identify whether children with DCD were different from normal children, in a systematic way.

Analysis for Hypothesis 3: Using standard techniques, the one-dimensional measure of normal gait was formed using the time/distance variables. Again, children with DCD were to be classified at least as abnormal or unusual using the time/distance covariance matrix for normal children. 


\section{CHAPTER 4}

\section{RESULTS}

The results of this study are presented in several sections. Screening for DCD and subject characteristics will be covered first, followed by Wilson's score using subjects with DCD, choosing one trial per subjects' side for children with DCD, testing the Fscore using young children; testing the Fscore using children with DCD; and finally, the results of the hypotheses tests.

\section{SCREENING FOR SUBJECTS WITH DCD}

A total of 333 grade one students from School District 18 were considered for inclusion in this study. A total of five schools participated, nominating 25 students ( $7.5 \%)$ considered to have difficulties with fine and/or gross motor skills. Of those 25 students, $16(4.8 \%)$ parental consent and health/activities questionnaires were returned correctly. Of those 16 children, 10 children fell at or below the $15^{\text {th }}$ percentile on the MABC Checklist (Henderson and Sugden, 1992), indicating at least moderate DCD. However, in some cases $(n=5)$, teachers wanted to proceed with the MABC Test, despite not scoring at or below the $15^{\text {th }}$ percentile, as the fine and/or gross motor skills were severely underdeveloped but not detected by the MABC Checklist. Therefore 15 ( 11 male, 4 female) children were screened using the MABC Test. A total of 11 subjects had scores at or 
below the $15^{\text {th }}$ percentile, representing $3.3 \%$ of the total population considered, and were contacted for participation in the gait analysis portion of this study.

Of the 15 children tested using the MABC, children 6 years of age $(n=10)$, had a mean MABC Checklist score of 67.6 , while children 7 years of age $(n=5)$ had a mean MABC Checklist score of 76.2. An average score of 18.09 ( $(\mathrm{d}=7.42)$ was found for the subjects who participated in the MABC Test $(n=15)$. Refer to Table 4.1 for details of the Movement $\mathrm{ABC}$.

Table 4.1

Results of the Movement $A B C$ for those Subjects Scoring Below the $15^{\text {th }}$ Percentile

\begin{tabular}{lcc}
\hline & Average Score & Standard Deviation \\
\hline MABC Checklist & 72.00 & 21.87 \\
MABC Test & 21.77 & 5.63 \\
$\quad$ Manual Dexterity & 10.36 & 2.81 \\
$\quad$ Bali Skills & 4.00 & 3.71 \\
$\quad$ Static and Dynamic & 7.59 & 2.30 \\
Balance & & \\
Note. $\mathrm{n}=11($ male $=9$, female $=2)$ &
\end{tabular}

\section{CHARACTERISTICS OF SUBJECTS WITH DCD}

A total of 11 subjects were invited to the Gait Laboratory at the University of New Brunswick. The gait patterns of seven subjects with DCD were recorded and analyzed. 
The DCD group (male $=6$, female $=1)$ had a mean age of 6.3 years $(s d=0.5)$ and a mean height of $120.1 \mathrm{~cm}(\mathrm{sd}=10.0)$. Please refer to Table 4.2 for the results of the MABC scores of the seven DCD subjects.

A health/activities questionnaire was filled out by each parent/guardian. The questionnaire asked questions regarding present health status, health history, and current activities and interests.

Table 4.2

Results of the DCD Group on the Movement ABC

\begin{tabular}{lccc}
\hline & Average & Subjects $<5^{\text {th }}$ & Standard \\
& Score & Percentile & Deviation \\
\hline MABC Checklist & 63.14 & 0 & 17.38 \\
MABC Test & 19.29 & 7 & 3.38 \\
$\quad$ Manual Dexterity & 10.00 & 6 & 3.19 \\
$\quad$ Ball Skills & 2.50 & 0 & 1.50 \\
$\quad$ Static and Dynamic & 6.77 & 2 & 2.29 \\
Balance & & & \\
\hline Note. $\mathrm{n}=7$ & & & \\
\hline
\end{tabular}

The seven subjects had no reported major medical problems, therefore a diagnosis of DCD could be given to all subjects. Six subjects were born at a full-term pregnancy, including one Cesarian section, while one subject was born prematurely. Average birth weight of the DCD group was 6 pounds, 12 ounces $(s d=2.95$ ). Six subjects reported no 
vision problems, while one subject wore glasses. Six subjects reported right-handedness and footedness, while one used the left hand and foot. The female subject reported that hospitalizations have occurred as seizures occur with a high fever, while none of the other subjects reported any major hospitalizations. There were no bone or joint problems with any of the subjects. Table 4.3 refers to reported major milestones during early childhood development. Many parents/guardians reported that their child was active in his/her play, however, only three subjects were involved in organized sports or activities. Hobbies and interests included activities such as bowling, soccer, movies, friends, and drawing.

Table 4.3

Reported Major Milestones during Earlv Development of the DCD Group

\begin{tabular}{lccc}
\hline \multicolumn{1}{c}{ Event } & $\mathrm{n}$ & Mean (Months) & Standard Deviation \\
\hline Sit & 5 & 5.2 & 1.26 \\
Crawl & 6 & 7.25 & 1.94 \\
Pull to Stand & 6 & 8.92 & 2.06 \\
Walk Alone & 7 & 11.5 & 2.5 \\
Run & 5 & 12.4 & 3.85 \\
\hline
\end{tabular}

\section{CHOOSING ONE TRIAL PER SUBJECTS' SIDE FOR CHILDREN WITH DCD}

The Vicon collected data on both the left and right sides, as suspected there may be different gait patterns between the left and right sides. Therefore one "typical" trial was chosen to represent each side, for each subject. This was in accordance with the methodology of the San Diego database, where a technician selected a "typical" cycle for 
each subject. Upon looking at the raw data, subjects had completed gait cycles ranging from 4-10 trials per side. Therefore it was decided that the "most typical" cycle would be chosen for that side.

In order to chose which trial was the "most typical" for that subject, the time/distance variables were chosen to average. Opposite toe off (OTO), single stance (SS), and toe off (TO) were selected as the variables to average. Opposite toe off (OTO) and toe off (TO) represented the percentage of the gait cycle where that event occurs. Whereas single stance (SS) is measured as the amount of time (calculated as a percentage of the gait cycle) spent on one limb. These three variables were chosen because they were included in the calculation of the Fscore developed in this study.

Each subject's values for each variable were placed in a row vector. Average values were calculated using all of the trials for that particular side for each subject. Equation 4.1 describes how the squared distance from the average was calculated.

Squared distance from the average $=($ vector - average $) \operatorname{Cov}^{-1}(\text { vector }- \text { average })^{\top}$

The vector is each subject's values for the three variables and the $\operatorname{Cov}^{-1}$ is the inverse covariance matrix calculated over all of the trials for that particular side for the subject. This type of equation has been the theme throughout this study (eq. $2.2,3.2$ ). The trial with the smallest number, or shortest squared distance from the average, was the trial chosen for the rest of the analysis for that side, for that subject. 


\section{WILSON'S SCORE USING SUBJECTS WITH DCD}

Using the selected trials, sagittal hip, knee, and ankle flexion/extension curves were investigated to classify normality using Wilson's (1998) methodology. Due to recording errors during data collection, many trials were discarded due to missing data (Appendix

I). Six trials remained with complete information for the sagittal hip, knee, and ankle flexion/extension curves and were transformed with Fourier coefficients and assigned a Wilson score. Table 4.4 lists the Wilson score results for each subject with the results of the MABC Test scores. Subjects DCD6 and DCD7 had both left and right sides included for the Wilson score. Opposing results were found for subject DCD6 whose left side was classified as abnormal and right was classified as normal. Interestingly, the two children classified by Wilson's (1998) technique as abnormal had the highest MABC Test scores, indicating the most severe cases of DCD.

Table 4.4

Wilson Scores for Subjects with DCD

\begin{tabular}{ccccl}
\hline ID & MABC Test Score & Side & $\begin{array}{c}\text { Wilson } \\
\text { Score }\end{array}$ & Classification \\
\hline DCD4 & 24.5 & $\mathrm{~L}$ & 19.01 & Abnormal \\
DCD5 & 16.5 & $\mathrm{R}$ & 0.86 & Normal \\
DCD6 & 19.5 & $\mathrm{~L}$ & 2.50 & Abnormal \\
DCD6 & 19.5 & $\mathrm{R}$ & 1.27 & Normal \\
DCD7 & 17.0 & $\mathrm{~L}$ & 0.52 & Normal \\
DCD7 & 17.0 & $\mathrm{R}$ & 1.22 & Normal \\
\hline
\end{tabular}


Wilson (1998) suggested looking at the 11-dimensional $\underline{B}^{(i)}$ vector (interpretable functions) for the abnormalities within the score. "The standardized vector describes 'standard deviations from the mean,' so that values above 2.0 or below -2.0 indicate unusual patterns" (Wilson, 1998, pg. 76). Wilson (1998) also indicated that an abnormal score may be given when none of the standardized $\underline{B}^{(i)}$ components are beyond \pm 2 , but all, or many are close to \pm 2 . Refer to Appendix $\mathrm{J}$ for the standardized $\underline{B}^{(t)}$ vectors for all 6 trials.

Taking a closer look at subject DCD6, the left side 11-dimensional standardized $\underline{B}^{(t)}$ vector was as follows:

standardized $\underline{\mathrm{B}}_{\mathrm{DCD}}=(-2.73,-1.46,-1.79,-0.13,1.20,0.41,0.67,0.34,-0.57,-0.94,0.46)$

The hip displacement value was the only component that was above +2 , however, ankle displacement was close to +2 , thereby giving an overall Wilson score of 2.5. Figure 4.1 illustrates the left hip displacement for this subject superimposed on the mean hip displacement from the normative database. 


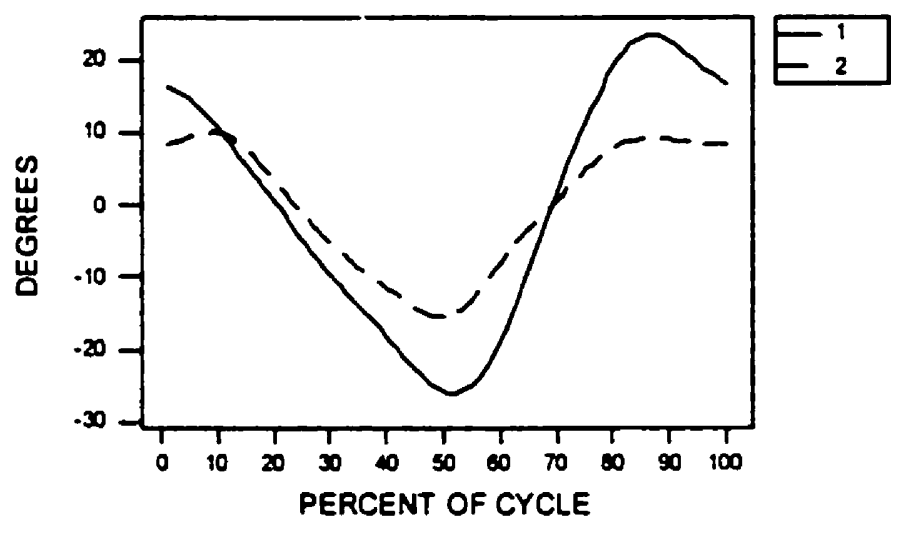

Solid line is men Curve

Figure 4.1 - Left Hip Displacement of Subject DCD6 and the Mean Hip Displacement Curve

Although DCD6 did not have a classification of abnormal on the right side, the 11-dimensional standardized $\underline{B}^{(i)}$ vector showed a large, -2.49 , component on hip displacement, which is seen in Figure 4.2. However, all other components were quite small, thereby resulting in a normal classification. As both sides indicated unusual patterns in hip displacement, it needs to be further investigated. 


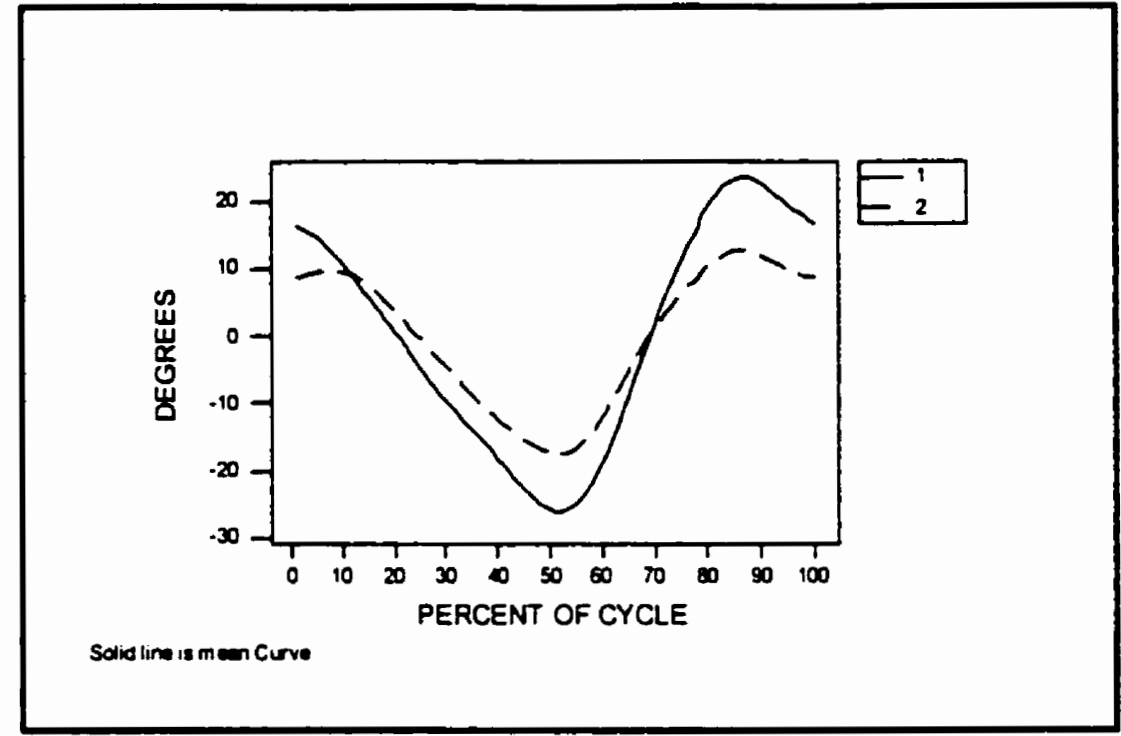

Figure 4.2 - Right Hip Displacement of Subject DCD6 and the Mean Hip Displacement Curve

Considering subject DCD4 with a score 19.01 the left sidel 1-dimensional standardized $\underline{B}^{(i)}$ vector was as follows:

standardized $\underline{B}_{D C D 4}=(-1.92,-2.80,-0.83,-2.16,0.84,-.097,-7.51,-0.91,0.07,1.68,-0.82)$

Another unusual pattern of hip movement could be seen in subject DCD4. Hip displacement (-1.92), hip velocity (-2.16), and hip acceleration (-7.51) were all unusual. Refer to Figures $4.3,4.4$, and 4.5 for a visual representation of the unusual patterns superimposed on the normal curves. The hip curve had four values ranging from - 70 to 60 , thereby altering the general shape of the graphs. Figure 4.6 shows knee displacement $(-2.80)$ for DCD4, with the unusual 'bump' at the same point in the cycle. 


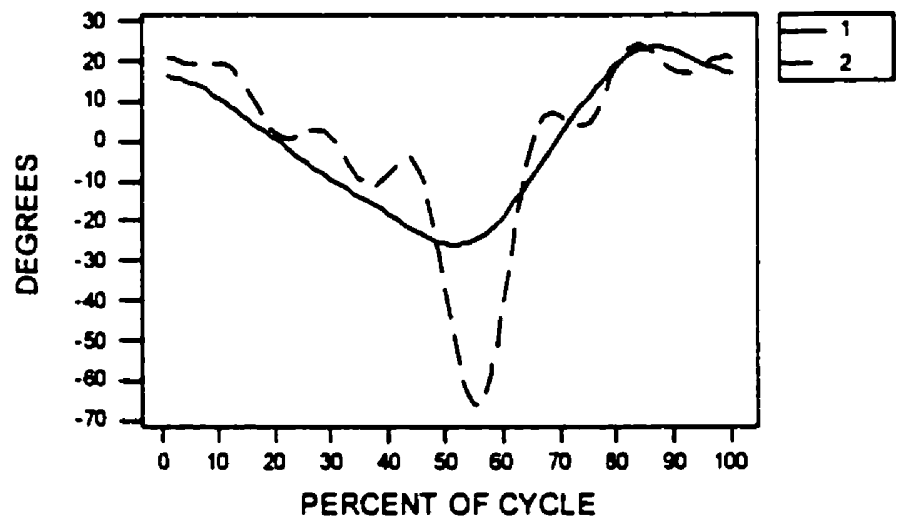

Solid line is mean Curve

Figure 4.3 - Left Hip Displacement of Subject DCD4 and the Mean Hip Displacement Curve

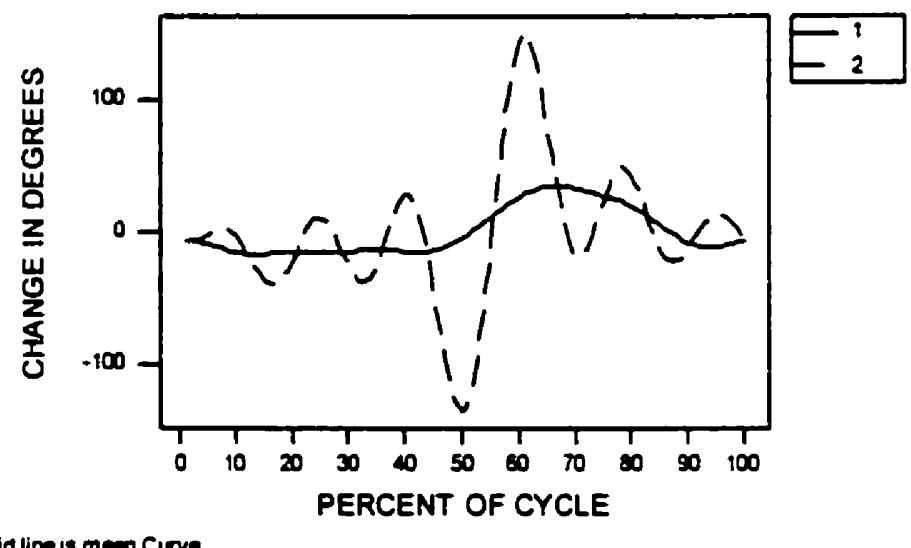

Figure 4.4 - Left Hip Velocity of Subject DCD4 and the Mean Hip Velocity Curve 


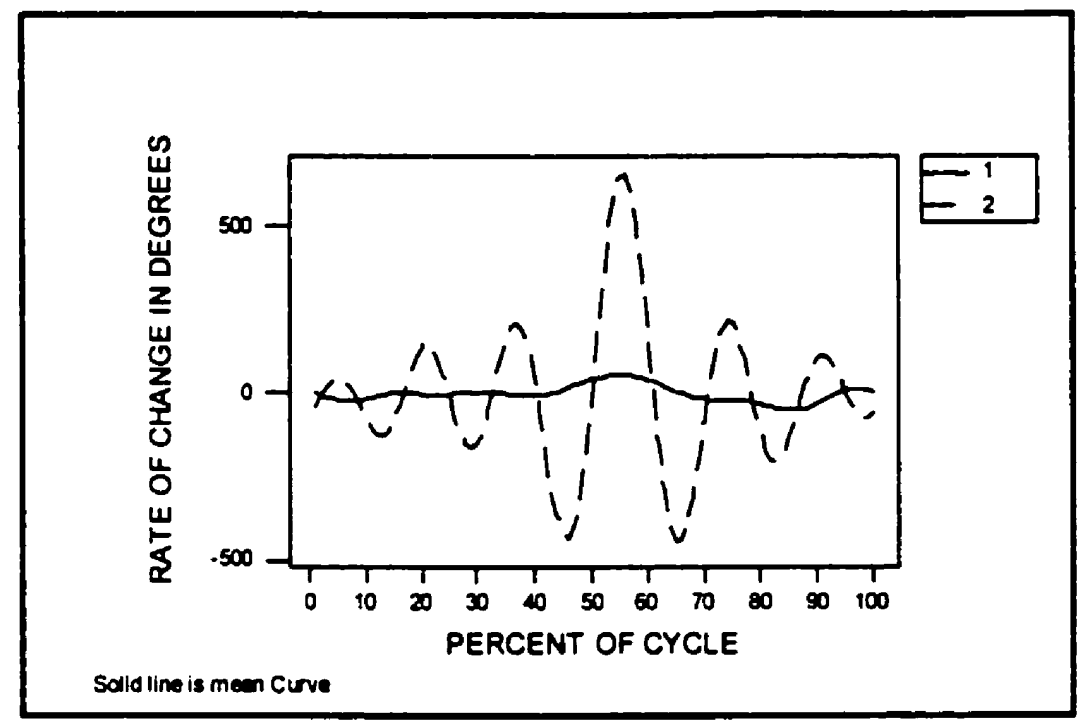

Figure 4.5 - Left Hip Acceleration of Subject DCD4 and the Mean Hip Acceleration Curve

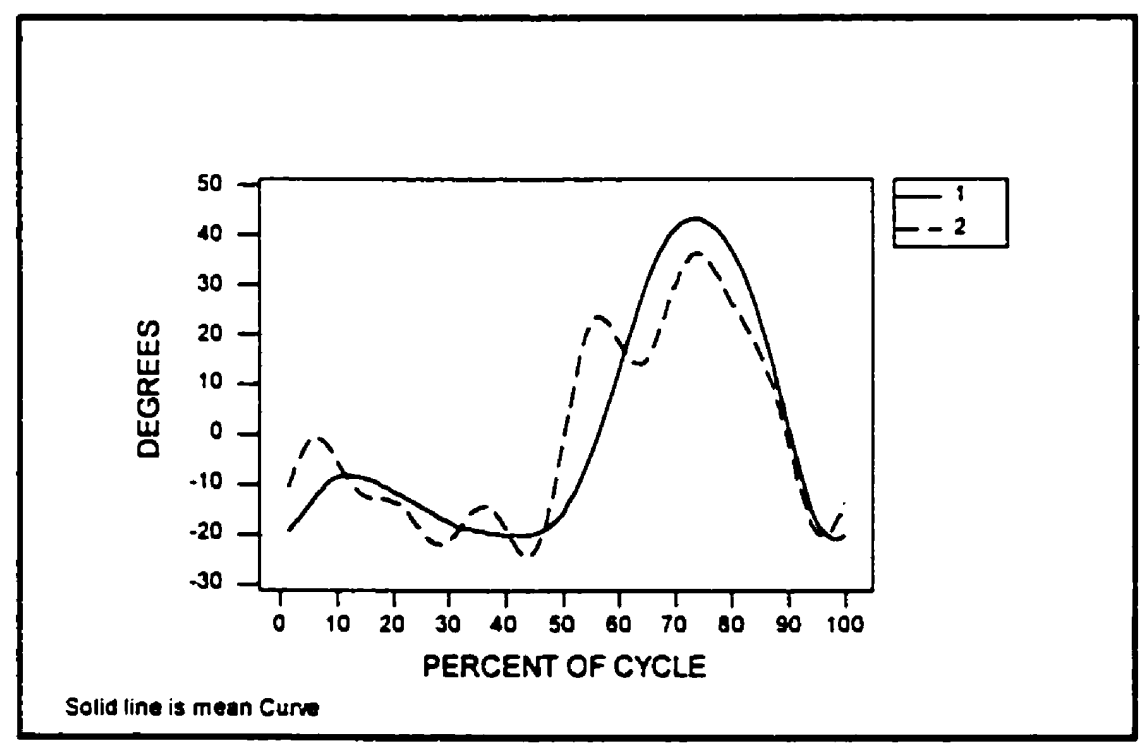

Figure 4.6 - Left Knee Displacement of Subject DCD4 and the Mean Knee Displacement Curve 


\section{TESTING THE FSCORE (YOUNG CHILDREN)}

The San Diego database was supplied, and only children three years and older were used to calculate the Wilson score and the Fscore calculated in this research. To test the Fscore, based on four time/distance variables, $F$ scores for the young children $(1,1.5,2$, and 2.5 years of age) were calculated. It is believed that children under three years of age do not have adult-like gait patterns. However, with increasing age, the gait patterns should become more normal. Refer to Table 4.5 for the results of the young children's Fscore, by age. As well, Table 4.6 includes both the classification results from the Wilson score and the Fscore, for the young children. It was interesting to note that the Fscore classified more children as abnormal than the Wilson score.

Table 4.5

Fscore Classification Results for Children less than Three Years of Age

\begin{tabular}{cccccccc}
\hline Age & $\mathrm{n}$ & Normal & & Unusual & \multicolumn{3}{c}{ Abnormal } \\
Group & & $(>2.26)$ & & $(2.26-2.69)$ & \multicolumn{3}{c}{$(<2.69)$} \\
\hline 1 & 96 & 12 & $13 \%$ & 3 & $3 \%$ & 81 & $84 \%$ \\
1.5 & 78 & 20 & $26 \%$ & 2 & $2 \%$ & 56 & $72 \%$ \\
2 & 88 & 39 & $44 \%$ & 4 & $5 \%$ & 45 & $51 \%$ \\
2.5 & 72 & 46 & $64 \%$ & 9 & $12 \%$ & 17 & $24 \%$ \\
\hline
\end{tabular}


Table 4.6

Classification Results of the San Diego Database for the Wilson score and the Fscore for Children Less Than Three Years old

\begin{tabular}{lcccr}
\hline & Wilson (1998) & \multicolumn{3}{c}{ Fscore $^{\mathrm{b}}$} \\
\hline Normal & 131 & $39 \%$ & 117 & $35 \%$ \\
Unusual & 52 & $16 \%$ & 18 & $5 \%$ \\
Abnormal & 148 & $45 \%$ & 199 & $60 \%$ \\
\hline
\end{tabular}

Note. ${ }^{a} n=331$ children. ${ }^{b} n=334$ children.

A larger number of children, 334, were classified using the Fscore as compared to the Wilson score, 331 , because time/distance data were available for more children.

Comparatively, $60 \%$ of the young children were classified as abnormal using the Fscore, as compared to $45 \%$ of the young children using the Wilson score. Interestingly, a larger number of young children were classified as unusual (16\%) using the Wilson score than the Fscore, reflecting the conservative nature of Wilson's (1998) cut-offs.

\section{TESTING THE FSCORE (SUBJECTS WITH DCD)}

The gait patterns of seven subjects with DCD were analyzed using the Fscore developed in this research. Both the left and right sides were grouped together (similar to the training set). In three cases, trials were omitted due to missing data, thereby leaving 11 complete trials to be analyzed. Table 4.7 includes the means and standard deviations of the four variables included in the Fscore for the DCD group compared with the San Diego 
training set. Table 4.8 includes the means and standard deviations of the other time/distance variables that were not included in the calculation of the Fscore for the DCD group compared with the San Diego training set. The averages were very similar between the two groups. Subjects with DCD consistently showed greater variation about the averages.

Table 4.7

Gait Characteristics of the DCD Group and San Diego Training Set for the Variables Included in the Fscore

\begin{tabular}{lcccccc}
\hline & \multicolumn{3}{c}{ DCD } & \multicolumn{3}{c}{ San Diego } \\
Characteristic & $\mathrm{n}$ & Mean & SD & $\mathrm{n}$ & Mean & SD \\
\hline Opposite Toe Off (\%) & 11 & 14.82 & 4.35 & 278 & 13.96 & 1.65 \\
Single Stance (\%) & 11 & 36.79 & 3.14 & 278 & 36.22 & 1.63 \\
Toe Off (\%) & 11 & 65.70 & 3.83 & 278 & 64.00 & 1.68 \\
Step Length (\%) & 11 & 49.58 & 6.55 & 278 & 50.01 & 1.24 \\
\hline
\end{tabular}


Table 4.8

Gait Characteristics of the DCD group and San Diego Training Set for the Variables Not Included in the Calculation of the Fscore

\begin{tabular}{lrrrrrr}
\hline & \multicolumn{3}{c}{ DCD } & \multicolumn{4}{c}{ San Diego } \\
Characteristic & n & Mean & \multicolumn{1}{c}{ SD } & n & Mean & SD \\
\hline Cycle Time (sec) & 11 & 0.84 & 0.09 & 139 & 0.81 & 0.08 \\
Cadence (step/min) & 11 & 145.04 & 13.88 & 139 & 150.09 & 13.88 \\
Stride Length (cm) & 11 & 80.15 & 13.66 & 139 & 81.94 & 11.01 \\
Walking Speed (cm/sec) & 11 & 97.08 & 18.96 & 139 & 102.3 & 15.99 \\
Opposite Foot Strike (\%) & 11 & 51.62 & 3.41 & 278 & 50.2 & 0.84 \\
\hline
\end{tabular}

Table 4.9 includes the classification of the 11 gait patterns that were analyzed using the Fscore. One trial was analyzed as normal and 10 trials as abnormal. The normal subject (DCD2) had only the left side analyzed. The four subjects whose left and right sides were analyzed were all classified as abnormal, indicating that they have similarly abnormal gait patterns on both left and right sides. The remaining two subjects had only one side analyzed and were both classified as abnormal. Refer to Table 4.4 for a comparison of the two gait scores. 
Table 4.9

Fscore Classification Results of the DCD Group

\begin{tabular}{llcl}
\hline Subject & Side & Score & Classification \\
\hline DCD1 & Left & $\mathrm{n} / \mathrm{a}$ & Omitted \\
& Right & 6.16 & Abnormal \\
DCD2 & Left & 2.24 & Normal \\
& Right & $\mathrm{n} / \mathrm{a}$ & Omitted \\
DCD3 & Left & 3.85 & Abnormal \\
& Right & $\mathrm{n} / \mathrm{a}$ & Omitted \\
DCD4 & Left & 3.03 & Abnormal \\
& Right & 59.26 & Abnormal \\
DCD5 & Left & 5.04 & Abnormal \\
& Right & 4.64 & Abnormal \\
DCD6 & Left & 2.81 & Abnormal \\
& Right & 7.76 & Abnormal \\
DCD7 & Left & 4.71 & Abnormal \\
& Right & 36.21 & Abnormal \\
\hline
\end{tabular}

\section{Results of Hypothesis}

The following section provides the results of this research as they apply to the hypothesis.

Hypothesis 1 stated that the one-dimensional measure of normality for the sagittal hip, knee, and ankle angle curves would classify most children with DCD as abnormal. Wilson's (1998) cut-off value was such that there was a $5 \%$ chance of classifying normal 
children as abnormal by chance. The sign test (using binomial calculations) was used to determine the probability of classifying two trials out of six as abnormal by chance. In this case, the Wilson score classified more DCD children as abnormal than one would have expected by chance $(\mathrm{p}$ value $=0.03)$. Therefore, Hypothesis 1 was accepted.

Hypothesis 2a stated that there would be differences in the means between normal children and children with DCD using the time/distance variables. Table 4.11 shows that this hypothesis was not born out by the data. As the variance structures were so different, multivariate analysis was not used. Instead, 2-sample t-tests were done on the four variables assuming unequal variances. Results indicated that there was no difference between the means of the normative database and the DCD subjects (OTO $p$ value $=0.52$, SS $p$ value $=0.56$, TO $p$ value $=0.17$, SL $p$ value $=0.83$ ). This indicated that there was no shift in the time/distance data between normal children and children with $\mathrm{DCD}$, as the results were all scattered around the same central position with subjects with DCD further away from the means (larger standard deviations). Therefore, Hypothesis $2 \mathrm{a}$ was rejected.

Hypothesis $2 b$ stated that there would be differences in the covariance structures between normal children and children with DCD using the time/distance variables. Appendix $\mathrm{K}$ gives both covariance and correlation matrices for the normative database and the DCD group. Differences were seen, at a glance, between the two groups. Referring to the diagonal entries (variances), children with DCD had more variation on all four measures. However, as Table J.2 has only 11 trials, generalizations must be made carefully. The largest difference between the two included groups was seen in step 
length. The step lengths of the DCD group had much stronger correlations with other variables as compared to normal children. Therefore, Hypothesis $2 \mathrm{~b}$ was accepted.

Hypothesis 3 stated that the new one-dimensional measure of normality based on the time/distance variables would classify most children with DCD as abnormal. The chosen cut-off was intended to classify $5 \%$ of normal children as abnormal by chance. The sign test (using binomial calculations) was used to determine the probability of classifying 10 out of 11 trials as abnormal by chance. The Fscore did classify most children with DCD as abnormal ( $p$ value $=0.00$ ) and perhaps did so better than the Wilson score. Therefore, Hypothesis 3 was accepted. 


\section{CHAPTER 5}

\section{DISCUSSION}

The discussion of the major findings from this study is preceded with discussions of screening for DCD and subject characteristics. Subsequently, the one-dimensional means of normality scores and the relationship between children with DCD and gait analysis will be discussed.

\section{SCREENING FOR DCD AND SUBJECT CHARACTERISTICS}

Subjects involved in the DCD group were included based on several factors. The subjects were originally nominated by their home room teacher. The MABC Checklist and Test were then completed on those children whom were nominated and had scores at or below the $15^{\text {th }}$ percentile.

Homeroom teachers were able to nominate children with fine and/or gross motor difficulties quite accurately. Six out of 16 subjects scored above the $15^{\text {th }}$ percentile on the Checklist, not warranting a diagnosis of DCD. However, in five of those cases, the teacher wanted to continue with the MABC Test as the fine/gross motor skills were noticeably underdeveloped. Four of those 5 subjects were diagnosed with severe DCD (all had scores greater than 17) on the MABC Test. Homeroom teachers do not see their students in the situations described by the MABC Checklist and had difficulty assigning a score for particular tasks. Many teachers noted that the scores, were a "best guess" situation. 
In one particular case, a teacher nominated a student with severely underdeveloped fine motor skills who scored 35 on the MABC Checklist, indicating no movement difficulties. However, the teacher requested that the child continue with the MABC Test, as the fine motor skills were so noticeably lacking compared to others in the classroom. The subject was also noted to be one of the best athletes in the school, participating in basketball, hockey, and soccer. Upon completion of the MABC Test, the final score did not warrant a diagnosis of DCD. However, the results of the fine motor section gave the poorest performance score, while showing no difficulties on any of the other sections. It is interesting to note that one of the largest groups of researchers in Canada studying DCD is working at the University of Western Ontario in the Occupational Therapy Department. Normally, a child is referred to this group on the basis of poor motor coordination, including poor handwriting (Martini and Palatajko, 1998). One would assume that in this case, the subject would have been referred to this clinic and would be currently receiving an intervention.

Sixteen students were tested using the MABC Test. Eleven of those students had scores at or below the $15^{\text {th }}$ percentile indicating DCD. Therefore, 11 of 333 students were diagnosed with DCD, representing $3.3 \%$ of the population. The Diagnostic and Statistical Manual - IV estimates that $6 \%$ of school age children between the ages of 5 and 11 have DCD (APA, 1994). Other studies (Henderson and Hall, 1982; Wright et al., 1994) have indicated approximately $5-16 \%$ of children having DCD. The prevalence of DCD in the present study was lower than indicated in the literature. 
Eleven subjects with $\mathrm{DCD}$ (nine male, two female) were invited to the Gait Laboratory at UNB. The gait patterns of seven subjects with DCD (six male, one female) were analyzed. In agreement with Piek and Edwards (1997) "a larger prevalence of DCD has been reported in boys than girls" (pg. 56). The findings of the present study are in agreement with the gender hypothesis.

Other characteristics of the DCD group included a mean height of $120.12 \mathrm{~cm}$ (sd $10 \mathrm{~cm}$ ), which is considered "normal" for children this age from Gallahue and Ozmun (1989, pg. $201 \&$ 202). Subject DCD4 was born premature, while all other subjects were born at a full term pregnancy. Prevalence of premature children among children with DCD has not been investigated by researchers, therefore, no conclusion can be made regarding the relationship between premature children and DCD. As well, subject DCD4 was the only subject who wore glasses, as he/she reported far-sightedness. A summary of reported major milestones during early development was presented in Table 4.3. Payne and Issacs (1991) suggested that independent walking is apparent by 12 months of age, even though normal is considered 9 to 17 months of age. The information reported by parents/guardians on the health/activities questionnaire was all within normal limits.

\section{ONE-DIMENSIONAL MEASURE OF NORMALITY SCORES}

\section{Wilson Score}

Due to unsuccessful data collection, missing data points were present in the trials for children with DCD. However, there was enough information to process six trials (three from the right side, three from the left side). Accordingly, two out of the six trials were 
classified as abnormal. Although not many of the trials of children with DCD were classified as abnormal, statistically, sign tests (using binomial calculations) indicated that more children than expected were classified as abnormal $(p$ value $=0.03)$. The Wilson score may not be as sensitive as expected, as children with DCD have abnormal gait patterns as seen in the Fscore.

\section{Fscore}

The one-dimensional measure of normality using the time/distance variables (Fscore) was calculated in this thesis. The San Diego database was provided and used to formulate the Fscore. A score using percentage of opposite toe off, percentage of single stance, percentage of toe off, and percentage of stride length was used. Biden, $O^{\prime}$ Connor, and Collins (1990) indicated that "measures of walking speed, cycle time, cadence, and step or stride length can be used to detect the slowing process. In the face of an injury or unstable limb, the usual response is to slow down and to favour the affected side" (pg. 298). As well, indications were made by Biden et al. (1990) "that measures of the relative percentage of single stance for each side and the timing of toe off and opposite foot strike can be used to determine the degree of gait symmetry for the subject" (pg. 298). As measures from both of these "categories" were included in the Fscore, there was a high level of confidence that the abnormalities would be detected.

The entire San Diego database was provided and only children three years and older were used to calculate the Fscore. The remaining young children were able to be used as a test case to ensure that the measure was classifying the gait patterns correctly. 
Referring to Table 4.9 , it is interesting to note that among 1.0 year old children, $84 \%$ were classified as abnormal as compared to $13 \%$ that were classified as normal. As the children aged ( 2.5 years of age) the gait patterns became more normal, as fewer children were classified as abnormal $(n=24 \%)$ and more children were classified as normal $(n=$ $64 \%)$.

In total, 11 gait trials (6 left side, 5 right side) for 7 subjects with DCD were tested using the Fscore. Ten out of 11 gait trials were classified as abnormal. It can be assumed that children with DCD do in fact have difficulties walking. Children with DCD typically have difficulty with more advanced gross motor skills such as running, hopping, skipping, and jumping. Independent walking is mastered before more advanced skills are learned. Results from this study indicated that walking patterns differed from normal children, at age six and seven.

The most unusual gait score results were seen with subjects DCD4 and DCD6, which are further described below. With the exception of subjects DCD4 and DCD6, all subjects followed a similar pattern of classification. Subject DCDI had an abnormal right side Fscore, while the other two scores were omitted due to missing data. Subject DCD2 had a normal left side Fscore, while the other two scores were omitted due to missing data. Subject DCD3 had an abnormal left side Fscore, while the other two scores were omitted due to missing data. Subject DCD5 had a normal right side Wilson score and abnormal left and right side Fscores. Subject DCD7 had normal left and right side Wilson scores while having abnormal Fscores on both the left and right sides. 


\section{SUBJECT DCD4}

The unusual Wilson score, 19.01 , on the left side indicated that there was something abnormal with the sagittal hip, knee, and ankle flexion/extension patterns. One explanation could lie in the missing data, as there were several missing data points at the beginning and end of some of the curves. Another possible explanation may be that the subject was different from other normal children. According to the standardized $\underline{B}^{(i)}$ vectors, problems lie within the hip curves (displacement $=-1.92$, velocity $=\mathbf{- 2 . 1 6}$, acceleration $=-7.51)$ as displayed in Figures 4.3, 4.4, and 4.5. Knee displacement $(-2.80)$ and the first prime frequency for mean angular knee displacement (1.68) also have large standard deviations from the means. The abnormal gait was noticed immediately by the primary researcher during data collection.

Subject DCD4 also yielded a Fscore of 59.26 for the right side and 3.03 for the left side. The Wilson score of 19.01 occurred on the left side, resulting in an unexpected classification for the Fscore on the right side. Noticeable differences on the right side were seen in percentage of opposite toe off $(26.23 \%)$, percentage of single stance (32.79 $\%)$, percentage of toe off (75.41\%), and percentage of single stance (63.64 \%) as compared to the means in Table 4.11. Left side data were closer to the mean than the right side, however considerable differences were still seen in percentage of opposite toe off ( $16.36 \%)$, percentage of single stance ( $34.55 \%)$, percentage of toe off $(67.27 \%)$, and percentage of single stance $(45.60 \%)$.

Subject DCD4 was seven years of age, had a height of $119 \mathrm{~cm}$, had a score of 44 on the MABC Checklist (above the $15^{\text {th }}$ percentile), and a score of 24.5 on the MABC 
Test ( $\left(1^{\text {st }}\right.$ percentile). This subject was born prematurely at 6 pounds, 2 ounces. Sitting occurred at 7 months, crawling at 10 months, pull to stand at 11 months, walking independently at 14 months, and running at 18 months were reported by the parent/guardian, which is all considered normal motor development. The subject was involved in several organized sports such as swimming, skating, and soccer. Interests and hobbies included animals, dancing, singing, and reading. Lack of movement experience would not be able to explain the movement difficulties of subject DCD4 as exposure to different movement patterns was evident in the many activities.

Interestingly, a high score of 13.5 was found on the manual dexterity portion of the MABC Test, indicating the $1^{\text {st }}$ percentile for that group of activities. The ball skills score of 4.0 fell in-between the $5^{\text {th }}$ and $15^{\text {th }}$ percentile and the static and dynamic balance score of 7.0 fell again, in-between the $5^{\text {th }}$ and $15^{\text {th }}$ percentile. These scores indicated problems over all three areas of the MABC Test. The largest degree of difficulty occurred with the fine motor skills portion of the MABC Test.

\section{SUBJECT DCD6}

Subject DCD6 had a left side Wilson score (2.50) high enough to warrant an abnormal classification, while the right side was considered normal. Both left and right side standardized $\underline{B}^{(t)}$ vectors indicated that hip displacement was more than \pm 2 standard deviations away from the mean. However, the left side had large knee displacement (-1.46), ankle displacement (-1.79), and knee velocity (1.20) standardized $\underline{B}^{(i)}$ vector components resulting in a Wilson score of 2.50 . In comparison, the right side 
standardized $\underline{\mathrm{B}}^{(n)}$ vector had only a large knee displacement component of 1.0 , which is not large enough to warrant a final score classification of abnormal. The two clashing descriptions indicated that DCD6 walked differently on the right side than on the left side, with abnormal hip displacement angle curve patterns on both sides.

Fscore values also gave abnormal classifications for both the left and right sides for subject DCD6. The left side Fscore was 2.81 and the right side Fscore was 7.76. Interestingly, different classifications were noted on the right side between the Wilson score and the Fscore. The Wilson score, 1.27, was normal and the Fscore, 7.76, was abnormal for the right side.

Subject DCD6 was 6 years of age, had a height of $129.5 \mathrm{~cm}$, had a score 50 on the MABC Checklist (above the $15^{\text {th }}$ percentile), and a score of 19.5 on the MABC Test ( $1^{\text {st }}$ percentile). This subject was born at a full term pregnancy weighing 8 pounds, 4 ounces. Sitting at 4.5 months, crawling at 5 months, pulling to stand at 5.5 months, walking independently and running at 8 months were reported by the parent/guardian. The subject was involved in no organized sports. Interests and hobbies included Pokemon, friends and movies.

Manual dexterity seemed to be the greatest difficulty for subject DCD6 resulting in a score of 12.5 on that MABC Test, indicating a $1^{\text {st }}$ percentile ranking for that group of activities. The ball skills score, 1.0 , fell above the $15^{\text {th }}$ percentile and a static and dynamic balance score, 6.0 , fell between the $5^{\text {th }}$ and $15^{\text {th }}$ percentile. The item scores, on the MABC Test, indicated problems primarily in the fine motor area with difficulty also occurring with static and dynamic balance. 
Subjects DCD4 and DCD6 did not have an MABC Checklist score that fell at or below the $15^{\text {th }}$ percentile. If the teachers had not wanted the two subjects to continue, they would not have been classified as having a severe case of DCD, and would not have participated in this research. Both of these subjects had the greatest amount of difficulty with the fine motor skills portion of the MABC Test, had unusual hip patterns based on the Wilson scores, and had abnormal classifications based on the Fscore.

\section{RELATIONSHIP BETWEEN SUBJECTS WITH DCD AND GAIT PATTERNS}

Gait patterns of children with DCD had not been researched, despite the lacking data. The results of this study indicated that children with DCD have abnormal gait patterns. Future investigations need to create a larger base of knowledge in this area. Several relationships could be made using the information that was generated by this study. Examples included walking was not strongly correlated with the walking component on the MABC Test, identification and intervention strategies could take on new dimensional, the Fscore was more sensitive than the Wilson score, and gait analysis could be used as a monitoring device of an intervention program.

Children included in this study were assessed with DCD using the MABC Test (Henderson and Sugden, 1992). There was one walking activity used for the MABC Test for each Age Band. Age Band I included walking with heels raised and Age Band II had children walking heel to toe, both along a 15 foot walkway. An earlier assumption was that there would be a correlation between the MABC Test walking component score and the two gait scores. However, there were no strong correlations between either of the 
gait scores and the score on the walking component of the MABC Test (Henderson and Sugden, 1992). The correlation of the walking component score on the MABC and the Fscore was $-0.426(p$ value $=0.191)$ and the correlation of the walking component score on the MABC and the Wilson score was $-0.107(p$ value $=0.840)$. Since both $p$-values were $>0.05$, the appropriate conclusion was that the walking component on the MABC Test score and the two gait scores were not correlated (as the correlation could well be zero). This could be due to the fact that the children had to walk a certain way (heels raised, heel to toe) for the MABC Test and not normal walking, which is how they were scored using the gait scores.

This research suggests abnormalities in gait patterns of children with DCD. This is significant in terms of guiding identification and intervention strategies. Identification processes and intervention strategies are two underdeveloped areas in the research of children with DCD. The Fscore was able to classify most children with DCD as abnormal, therefore researchers, educators and clinicians could use this type of qualitative measurement to diagnose and screen for DCD in children. Clinicians could use the information of abnormal walking patterns to develop new intervention strategies. Walking pattern instruction could be used to determine if walking could aid children with more efficient running, hopping, jumping, and kicking movement patterns.

Interestingly, the Fscore classified more children's gait patterns as abnormal than the Wilson score. The variables required to calculate the Fscore are easier to measure than those for the Wilson score. The simplicity of the measurement can be seen with the number of trials that each score was able to process. The Wilson score was only able to 
use six trials, as there was missing data points with the other eight trials. The Fscore was able to process 11 trials, while the remaining 3 trials had missing data. Simple video tape analysis would have been suitable for this type of calculation. Clinicians and educators who are working with children that may have movement difficulties could easily use this type of classification. One could go into the school with only a video camera to record the gait patterns of numerous children, record the time/distance variables, input those variables into a simple computer program, and be able to identify children with DCD. Another possibility for researchers and clinicians of the Fscore is using it as a monitoring device for an intervention program. The effectiveness of an intervention program could explore if there is a learning effect from the gait analysis procedure. Researchers have commented on the difficulty of assessing an intervention program due to a transfer effect of testing. Gait analysis could be one solution, as individuals perform many gait cycles on any given day. The intervention program would have to include proper walking instruction so that the child would not be "practising" abnormal walking, thus creating a bad habit. However, the Fscore would easily detect changes in the gait patterns as the score would decrease (become closer to the mean) to show that the movement pattern is becoming more normal. 


\section{CHAPTER 6}

\section{SUMMARY, CONCLUSIONS, AND FUTURE RECOMMENDATIONS}

\section{SUMMARY}

The purpose of this study was to validate Wilson's (1998) statistical procedure of classifying the gait patterns of a population, $D C D$, that may not be so abnormal using naked eye observation. Wilson's one-dimensional measure of normality (Wilson score) used sagittal hip, knee, and ankle flexion/extension data to classify the gait pattern as normal, unusual, or abnormal. A secondary objective of this research was to formulate another one-dimensional measure of normal gait (Fscore) using the time/distance variables, as more often these variables are easier and less expensive to measure. The statistical procedures were formulated using gait data on normal children from the San Diego Children's Hospital study by Sutherland et al. (1988). The Fscore would be easier for clinicians and educators to use for diagnostic and treatment purposes.

Children with DCD were chosen as the clinical population as they generally have difficulties with fine and/or gross motor skills. Gait abnormalities of children with DCD were assumed to be less apparent to the naked eye than gait abnormalities of children with Downs Syndrome or Hypotonia. As Revie and Larkin (1993) indicated, "children who look awkward and are described as clumsy, dyspraxic, or poorly coordinated have difficulty acquiring and performing basic movement patterns such as walking, running, hopping, jumping, throwing, catching, kicking, and hitting" (pg. 29). Therefore, children 
with DCD are an ideal population to determine the power of Wilson's (1998) statistical procedure.

A total of 333 children, from 5 schools, were considered for the DCD group. Homeroom teachers nominated and filled out the MABC Checklist on those students who they thought may have problems with fine and/or gross motor abilities. The MABC Test was administered to 16 subjects for whom the teacher had nominated and parental consent was obtained. A total of 11 subjects were diagnosed with $D C D$ using the MABC Test and invited to the Gait Laboratory at UNB.

The Fscore was formulated using gait data on 178 normal children (aged $3-7$ years old) included in the San Diego database (Sutherland et al., 1988). The Fscore used four measures; percentage of opposite toe off, percentage of single stance, percentage of toe off, and step length as a percentage of stride length. The Fscore was tested using data on normal young children (aged I - 2.5 years old) from the San Diego database, which was similar to Wilson's (1998) methodology. The Fscore of young children was consistent with gait pattern characteristics of young children and discriminated well between normal and abnormal gait patterns.

The gait patterns of seven subjects with DCD (six male and one female) were analyzed using the two independent gait scores. Both the Wilson score and the Fscore classified most children with DCD as abnormal. The following includes the important findings from this study, as well as a list of recommendations for future studies in the area. 
1. The Movement $A B C$ was effective in diagnosing children with DCD in five schools of District 18 , as approximately $7.5 \%$ of the grade one population was nominated as having difficulty with fine and/or gross motor skills. Diagnosis of DCD was made to $3.3 \%$ of the grade one population using the MABC.

2. The standing heights and reported major developmental milestones suggested that the physical development of children with DCD was not generally delayed or different from children classified as normal.

3. More children with DCD were classified as having abnormal gait using Wilson's (1998) one-dimensional measure of normality using sagittal hip, knee, and ankle curves than had been expected.

4. On average, children with DCD did not differ from normal children with respect to individual time/distance gait variables (percentage of opposite toe off, percentage of single stance, percentage of toe off, and step length as a percentage).

5. Children with DCD and normal children had different covariance structures based on time/distance measurements, and children with $\mathrm{DCD}$ tended to vary greatly from average values of normal children.

6. Most children with DCD were classified as abnormal using the Fscore developed in this research.

\section{CONCLUSION}

The important result from this study indicated that children with DCD had abnormal gait patterns, which was identified using the Fscore. Sign tests (using binomial calculations) 
indicated that the Wilson score classified more children with DCD as abnormal than had been expected and the Fscore classified most children with DCD as abnormal. The Wilson score classified 2 out of 6 trials as abnormal while the Fscore classified 10 out of 11 trials as abnormal. Therefore, the Fscore was able to classify more children with DCD as abnormal.

As children with DCD typically have difficulty with gross motor skills such as running, hopping, jumping, and kicking, it is interesting to note the difficulty that they have with the first learned movement pattern, walking. Gait patterns have been widely studied in different populations of children and typically any deviation from the mean indicates an abnormality. As both of the gait scores indicated abnormalities with children with $D C D$, identification and intervention strategies have new possibilities. Specifically, research needs to concentrate on the time/distance measures as the Fscore classified more children as abnormal than Wilson's score.

\section{RECOMMENDATIONS}

Based on the current status of research and results from this study, the following recommendations are offered.

1. Cross-sectional and longitudinal studies are required to determine if the abnormal gait patterns identified in this thesis are consistent with age.

2. Determine if other gross motor skills of children with DCD would be classified as abnormal compared to normal children. 
3. Determine if other quantitative measures (gait, running, hopping, skipping, jumping) show similar correlation patterns with qualitative measurements (items on the MABC Test).

4. As both of the scores were formulated using the same normative database, it would be useful to test these scores on another normal population to determine if they are being classified correctly.

5. Future investigations should study other special populations using Wilson's score and the Fscore.

6. Formulate a new diagnostic technique and test on children with $D C D$ validating the diagnostic power against the MABC Test.

7. Formulate an intervention strategy based on abnormal gait patterns and validate with children with DCD.

8. Using the Fscore calculation as a monitoring device of an intervention program, determine the effectiveness of that intervention program and explore if there is a learning effect from the gait analysis procedure.

9. Future investigations should study the relationship between premature births as a risk factor for DCD. 


\section{REFERENCES}

American Psychiatric Association. (1994). Diagnostic and Statistical Manual (DSM-IV) (4th ed.). University of Western Ontario: Developmental Coordination Disorder Web Site.

Armitage, M. \& Larkin, D. (1993). Laterality, motor asymmetry and clumsiness in children. Human Movement Science, 12, 155-177.

Ayyappa, E. (1997). Normal human locomotion, part 1: Basic concepts and terminology. Journal of Prosthetics and Orthotics, 9, (1), 10-17.

Biden, E., O'Connor, J., \& Collins, J. (1990). Gait analysis. In D. Daniel et al. (Eds.), Knee ligaments: Structure, function, injury and repair (pg. 291-311). Raven Press, Ltd.

Bischoff J.A. \& Lewis, K.A. (1987). A cross-sectional study of fitness levels in a movement education program. Research Quarterly for Exercise and Sport. 58 (3), 348353.

Bouffard, M., Watkinson, E., Thompson, L., Causgrove Dunn, J. \& Romanow, S. (1996). A test of the activity deficit hypothesis with children with movement difficulties. Adapted Physical Activity Quarterly, 13, 61-73.

Caine, D.J. (1990). The U.N.B. motor development clinic for young children. CAHPER Journal, 56 (6), 36-37.

Cantell, M., Ahonen, T. \& Smyth, M. (1994). Clumsiness in Adolescence: Educational, motor, and social outcomes of motor delay detected at 5 years. Adapted Physical Activity Quarterly, 11, 115-129. 
Causgrove Dunn, J. \& Watkinson, E. (1996). Problems with identification of children who are physically awkward using the TOMI. Adapted Physical Activity Quarterly, 13, 347-356.

Dare, M. \& Gordon, N. (1970). Clumsy children: A disorder of perception and motor organization. Developmental Medicine and Child Neurology, 12, 178-185.

Deluzio, K.J. (1997). Modelling and Analysis of Gait Waveforms. Unpublished master's thesis, Queens University, Kingston, Ontario, Canada..

Deluzio, K.J., Wyss, U.P., Zee, B., Costigan, P.A., \& Sorbie, C. (1997). Principal component models of knee kinematics and kinetics: Normal vs. pathological gait patterns. Human Movement Science, 16, 201-217.

Developmental Coordination Disorder: Twenty Questions. School of Occupational Therapy, Faculty of Health Sciences, The University of Western Ontario Homepage.

Dewey, D. \& Kaplan, B. (1992). Analysis of praxis task demands in the assessment of children with developmental motor deficits. Developmental Neuropsychology, 8 (4), 367-379.

Dowsett, B. (1995, Spring). Clumsy kids can. Alumni, Western Gazette, 20-21. Fox, A. Clumsiness in Children. University of Western Ontario: Developmental Coordination Disorder Webpage.

Fox, A. \& Lent, B. (1996). Clumsy children: Primer on developmental coordination disorder. Canadian Family Physician, 42, 1965-1971. 
Gallahue, D.L. \& Ozmun, J.C. (1989). Understanding motor development: Infants, children, adolescents, adults ( $3^{\text {rd }}$ ed. ). Madison, WI: Brown \& Benchmark.

Geuze R. \& Borger, H. (1993). Children who are clumsy: Five years later. Adapted Physical Activity Quarterly, 10, 10-21.

Hall, S.J. (1995). Basic Biomechanics ( $2^{\text {nd }}$ ed.). St. Louis, MO: Mosby.

Haywood, K.M. (1986). Lifespan motor development. Champaign, IL: Human Kinetics.

Health Canada \& Canadian Society for Exercise Physiology. (1998). Canada's Physical Activity Guide to Healthy Active Living (No. H39-429/1998-1E ISBN 0-66286627-7). Ottawa, Canada.

Henderson, S. (1987). The assessment of clumsy children: New and old approaches. Journal of Child Psychology and Psychiatry, 28 (4), $511-527$.

Henderson, S. (1994, October). The Natural History and Long Term Consequences of Clumsiness in Children. Paper presented at the conference Children and Clumsiness: a Disability in Search of Definition, London, Ontario.

Henderson, S. \& Hall, D. (1982). Concomitants of clumsiness in young school children. Developmental Medicine and Child Neurology, 24, 448-460.

Henderson, L., Rose, P. \& Henderson, S. (1992). Reaction time and movement time in children with a developmental coordination disorder. Journal of Child Psychology and Psychiatry, 33 (5), 895-905.

Henderson S.E. \& Sugden, D.A. (1992). Movement assessment battery for children. London, UK: The Psychological Corporation, Harcourt Brace and Company. 
Hoare, D. (1994). Subtypes of developmental coordination disorder. Adapted Physical Activity Quarterly, 11, 158-169.

Howard, L. (1997). Developmental coordination disorder: Can we measure our intervention? British Journal of Occupational Therapy, 60 (5), 219-221.

Hsu, E., Bardfield, S., Cratty. \& Garfinkel, A. (1989). Cinematographical methods to assess associated movements in children. Adapted Physical Activity Quarterly, 6, 255-267.

Jacobson, R. (1998). Approach to the child with weakness or clumsiness. Pediatric Clinics of North America, 45 (1), 145-168.

Knuckly, N. \& Gubbay, S. (1983). Clumsy children: A prognostic study. Australian Pediatrician Journal, 19, 9-13.

Leemrijse, C., Meijer, O.G., Vermeer, A., Lambregts, B \& Ader, H. (1999). Detecting individual change in children with mild to moderate motor impairment: the standard error of measurement of the movement ABC. Clinical Rehabilitation, 13, 420. 429.

Lord, R. \& Hulme, C. (1987). Perceptual judgements of normal and clumsy children. Developmental Medicine and Child Neurology, 29, 250-257.

Losse, A., Henderson, S., Elliman, D., Hall, D., Knight, E. \& Jongmans, M. (1991). Developmental Medicine and Child Neurology, 33, 55-68.

Maeland, A. (1992). Identification of children with motor coordination problems. Adapted Physical Activity Quarterly, 9 , 330-342. 
Majnemer, A. \& Shevell, M. (1995). Diagnostic yied of the neurologic assessment of the developmentally delayed child. Journal of Pediatrics, (August), 193199.

Marchiori, G., Wall., A. \& Bedingfield, W. (1987). Kinematic analysis of skill acquisition in physically awkward boys. Adapted Physical Activity Quarterlv, 4, 305315.

Martini, R. \& Polatajko, H.J. (1998). Verbal self-guidance as a treatment approach for children with developmental coordination disorder: a systematic replication study. The Occupational Therapy Journal of Research, 18 (4), 157-181.

Minitab Student Version Release 12 for Windows [Computer Software]. (1998). Pacific Grove, California: Brooks/Cole Publishing Company.

Missiuna, C. (1994). Motor skill acquisition in children with developmental coordination disorder. Adapted Physical Activity Quarterly, 11, 214-235.

Missiuna, C. \& Pollock, N. (1995). Beyond the norms: Need for multiple sources of data in the assessment of children. Physical and Occupational Therapy in Pediatrics, 15 (4), 57-71.

Miyahara, M., Tsujii, M., Hanai, T., Jongmans, M., Barnett, A., Henderson, S., Hori, M., Nakanishi, K. \& Kageyama, H. (1998). The movement assessment battery for children: A preliminary investigation of its usefulness in Japan. Human Movement Science, 17, 679-697.

O’Beirne, C., Larkin, D. \& Cable, T. (1994). Coordination problems and anaerobic performance in children. Adapted Physical Activity Quarterly, 11, 141-149. 
Payne, V.G. \& Isaacs, L.D. (1991). Human motor development, A lifespan approach. Mountain View, CA: Mayfield Publishing Company.

Piek, J. \& Coleman-Carman, R. (1995). Kinesthetic sensitivity and motor performance of children with developmental co-ordination disorder. Developmental Medicine and Child Neurology, 37, 979-984.

Piek, J. \& Edwards, K. (1997). The identification of children with developmental coordination disorder by class and phsyical education teachers. British Journal of Educational Psychology, 67, 55-67.

Reuben, R. \& Bakwin, H. (1968). Developmental clumsiness. Pediatric Clinics of North America, 15 (3), 60 I-610.

Revie, G \& Larkin, D. (1993). Task-specific intervention with children reduces movement problems. Adapted Physical Activity Quarterly, 10, 29-41.

Riggen, K.J., Ulrich, D.A. \& Ozmun, J.C. (1990). Reliability and concurrent validity of the test of motor impairment - Henderson revision. Adapted Physical Activity Quarterly, 7, 249-258.

Rosblad, B. \& Gard, L. (1998). The assessment of children with developmental coordination disorder in Sweden: A preliminary investigation of the suitability of the movement ABC. Human Movement Science, 17, 711-719.

Reuben, R.N. \& Bakwin, H. (1968). Developmental clumsiness. Pediatric Clinics of North America, 15, (3), 601-610). 
Schoemaker, M., Hijlkema, M. \& Kalverboer, A. (1994). Physiotherapy for clumsy children: An evaluation study. Developmental Medicine and Child Neurology, $\underline{36}, 143-155$.

Schoemaker, M. \& Kalverboer, A. (1994). Social and affective problems of children who are clumsy: How early do they begin? Adapted Physical Activity Quarterly, 11, 130-140

Sigmundsson, Pedersen, Whitting \& Ingvaldsen. (1998). We can cure your child's clumsiness! A review of intervention methods. Scandinavian Journal of Rehabilitation Medicine, 30, 101-106.

Skorji, V. \& McKenzie, B. (1997). How do children who are clumsy remember modelled movements? Developmental Medicine and Child Neurology, 39, 404-408.

Smits-Engelsman, B., Henderson, S. \& Michels, C. (1998). The assessment of children with developmental coordination disorder in the Netherlands: The relationship between the movement assessment battery for children and the korperkoordinations test fur kinder. Human Movement Science, 17, 699-709.

Smyth, M. \& Mason, U. (1997). Planning and execution of action in children with and without developmental coordination disorder. Journal of Child Psychologv and Psychiatry, 38 (8), 1023-1037.

Steinwender, G., Saraph, V., Scheiber, Zwick, E. B., Uitz, C. \& Hackl, K. (2000). Intrasubject repeatability of gait analysis data in normal and spastic children. Clinical Biomechanics, 15, 134-139. 
Sutherland, D.H., Olshen, R.A., Biden, E.N. \& Wyatt, M.P. (1988). The development of mature walking. Oxford, UK: Mac Keith Press.

Ulrich, R. (1985). Test of Gross Motor Development. Austin, Texas: Pro-Ed.

Whittle, M. (1991). Gait analysis: An introduction. Oxford, UK: ButterworthHeinemann Ltd.

Wilson, C.D. (1998). An analysis of the patterns of variation in the gait of normal children aged three to seven. Unpublished master's thesis, University of New Brunswick, Fredericton, New Brunswick, Canada.

Wilson, P. \& McKenzie, B. (1998). Information processing deficits associated with developmental coordination disorder: A meta-analysis of research findings. $\underline{\text { Journal }}$ of Child Psychology and Psychiatry, 39 (6), 829-840.

Wright, H. (1997). Children with developmental co-ordination disorder - a review. European Joumal of Physical Education, 2, 5-22.

Wright, H. \& Sugden, D. (1996a). The nature of developmental coodination disorder: Inter- and intragroup differences. Adapted Physical Activity Quarterlv, 13, 357371.

Wright, H. \& Sugden, D. (1996b) A two-step procedure for the identification of children with developmental co-ordination disorder in Singapore. Developmental Medicine and Child Neurology, 38, 1099-1105.

Wright, H., Sugden, D., Ng, R. \& Tan, J. (1994). Identification of children with movement problems in Singapore: Usefulness of the movement ABC checklist. Adapted Physical Activity Quarterly, 11, 150-157. 
Appendix A: Health/Activities Questionnaire 


\section{HEALTH/ACTIVITIES QUESTIONNAIRE}

Name

Address

Date of birth

Phone Number

Parent's Name

\section{PAST HISTORY}

Born:

at term/premature

Sex:

male/female

Birthweight

Difficulties at birth

Any chronic medical conditions

Wears glasses

$$
\text { yes/no }
$$

Handedness/Footedness:

$\begin{array}{ll}\text { Eat } & \text { right/left } \\ \text { Write } & \text { right/left } \\ \text { Throw } & \text { right/left } \\ \text { Kick } & \text { right/left }\end{array}$

Any family history of:

Congenital dislocation of hip

yes/no

Club foot

yes/no

Rotational problems of lower limbs

yes/no

Severe bow legs

yes/no

Severe knock knees

yes/no 
Any major injury of illness requiring hospitalization

yes/no

if yes, please explain

Any fracture, dislocation or other bone or joint disease yes/no

if yes, please explain

\section{DEVELOPMENTAL MILESTONES}

At which age did he/she first:

Sit?

Crawl?

Pull to stand?

Walk alone?

Run?

\section{PRESENT HEALTH STATUS}

Any illness at present? yes/no

if yes, please explain

Any problems with vision? yes/no

if yes, please explain

\section{ACTIVITIES}

Does your child participate in any organized sports or activities?

if yes, please elaborate (amount of time per week)

What are your child's interests and hobbies? 
Appendix B: Gait Laboratory Diagram 


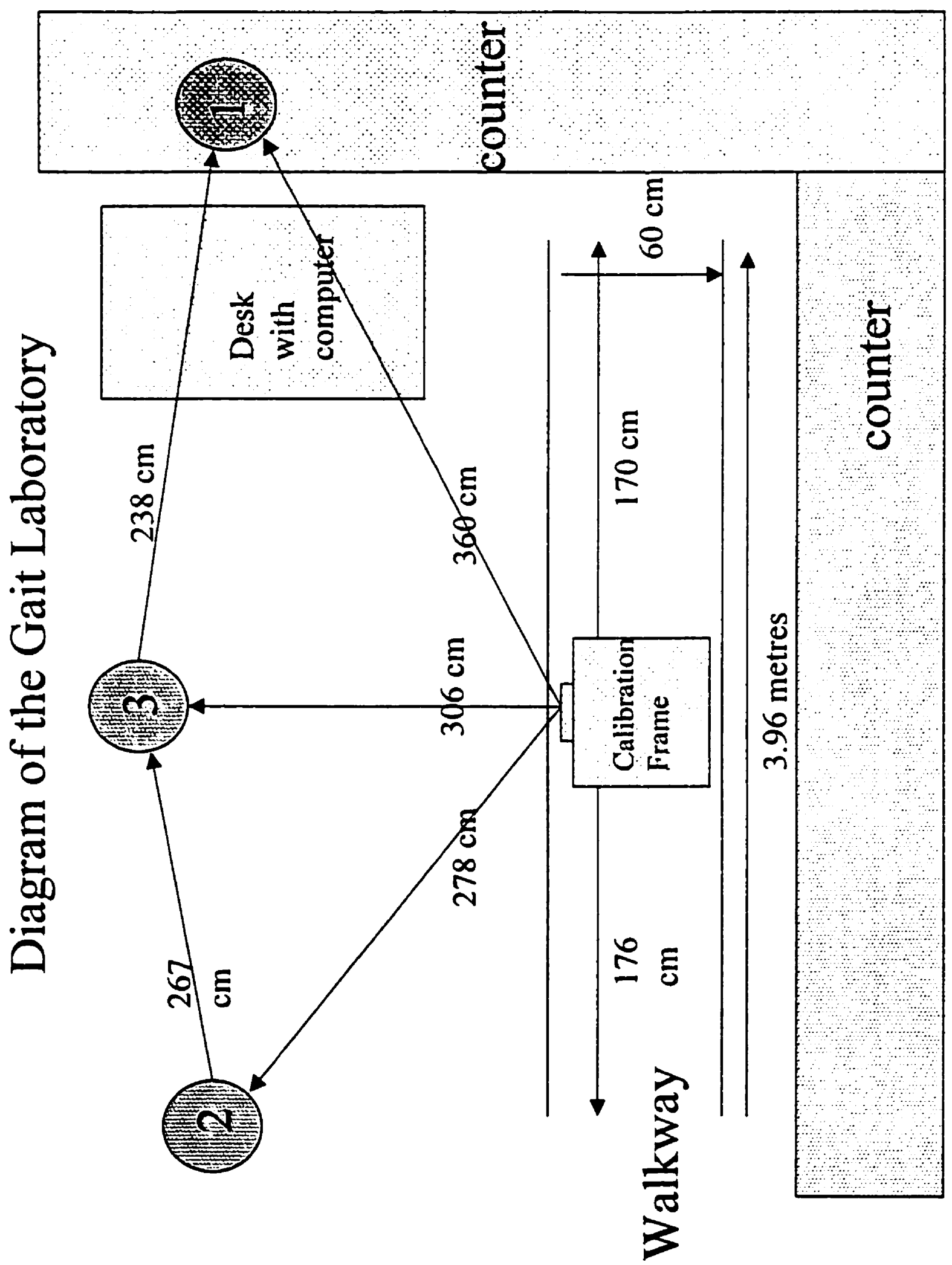


Appendix C: School District 18 Approval 


\section{Nouveau}

January 31,2000

Ms. Connie Bothwell-Myers

Associate Professor

Faculty of Kinesiology

University of New Brunswick

P. O. Box 4400

Fredericton, NB

E3B 5 A3

Connić

Dear Ms Bottiwell-Myers:

Permission is granted for you to conduct your proposed study, with your graduate student, Sarah Woodruff, with grade one students experiencing movement difficulty in School District 18.

I understand that several revisions to your original proposal have been agreed upon to ensure that the project can be carried out in the most effective manner possible. I hope that by focusing the study on three schools, that your target dates can be met and the research successfully concluded.

I look forward to reviewing the results of your survey.

Yours truly,

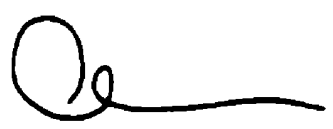

Alex Dingwall

Director of Education

$A D / j m b$

cc Zoë Watson

Gary Harding

Steve Pierce

Reg Bonnell

Tel.Telephune

(fiki) +isisit

Fax/Telecupreur

$(516)+73-230$ qoi Presumin it

P.) Bux 10

Fredencton

ien Brunswick

Candus ESB trit
565. rue Prestmin

Cise postise :I!

Fredencturn

Siluveau-Brunswich

Ciniales E:B +Yit 
Appendix D: Kinesiology Ethical Approval Form 


\section{University of New Brunswick \\ FACULTY OF KINESIOLOGY}

\section{Certificate of Ethical Acceptablility of Research and Other Studies Involving Human Participants}

This is to certify that the Faculty of Kinesiology Ethics Review Committee has examined the research proposed or other type of study submitted by:

\begin{tabular}{|l|l|}
\hline Principal Investigator / Supervisor & Connie Bothwell-Myers \\
\hline Student Investigators & Sarah Woodruff \\
\hline Entitled & Gait Patterns of Children with DCD \\
\hline
\end{tabular}

and concludes that in all respects the proposed project meets appropriate standards of ethical acceptability.

\section{Members of Ethics Committee}

\begin{tabular}{|l|c|c|}
\hline \multicolumn{1}{|c|}{ Name (printed) } & Signature & \multicolumn{1}{c|}{ Date } \\
\hline \hline Shirley Cleave & & \\
\hline Ian Reid & & \\
\hline Chris Stevenson & & March 21, 2000 \\
\hline
\end{tabular}

Comments (if any): 
Appendix E: Letter to Parents and Parental Consent Form 
February 23, 2000

Dear Parents/Guardians,

We are writing to invite your son/daughter to participate in a research study being conducted through the University of New Brunswick. This research project has received approval from the Director of Education for District 18 and from the principal of your child's school.

Please read all information and fill out the parental consent and health/activities questionnaire as soon as possible. If you have any questions please do not hesitate to contact Sarah Woodruff (455-4348) or Dr. Connie Bothwell-Myers (453-5035 or cbm@unb.ca).

The purpose of this study is to validate a statistical procedure used to determine the degree of maturity of walking patterns in children. The procedure has already been tested on children that have obvious differences in walking patterns. Children, from grade 1 classrooms, that may have difficulty with fine motor (ex. handwriting) and/or gross motor (ex. running) skills have been selected to be included in this study.

There are two different steps that your child will undergo. First, the researcher will look at the fine and gross motor skills of your child using the MABC Test. Observations include items such as manual dexterity, ball skills, walking and static and 
dynamic balance. This is done to assess the nature of his/her fine and gross motor abilities.

Once your child's general motor skills have been assessed, he/she will be invited to the Gait Laboratory at the University of New Brunswick. He/she will be asked to walk along a walkway several times while being videotaped. Your child will be asked to wear a bathing suit so that reflective markers can be placed on the body. This portion of the study will take approximately one hour and you are encouraged to stay and watch.

Finally we ask that you fill out the attached consent form and health/activities questionnaire. Your responses on the health/activities questionnaire will provide a complete picture of your child's past medical history and current interests.

Should you decide that your child could benefit from a movement education/enrichment program, the researchers are able to facilitate such a program through the Faculty of Kinesiology at the University of New Brunswick. Please complete the attached portion of this consent form and return it to school with your child. If you have any questions or concerns regarding this research project, please feel free to contact Sarah Woodruff (455-4348) or Dr. Connie Bothwell-Myers (453-5035 or cbm@unb.ca). Thank your very much for your time.

$$
\text { Sincerely, }
$$

Dr. Connie Bothwell-Myers

Dr. Maureen Tingley 


\section{PARENTAL CONSENT FORM}

I understand that this research project is being conducted through the Faculty of Kinesiology at the University of New Brunswick to validate the discriminating power of a statistical technique using children in grade 1 .

I understand that my child will be assessed using the Movement $\mathrm{ABC}$ to determine the level of mastery of his/her movement skills.

I understand that my child will be invited to the Gait Analysis Laboratory at the University of New Brunswick to facilitate the videotaping of a specific movement pattern (walking).

I understand that my child's results on all of these tests will be completely confidential. Only the researchers and research assistants will have access to the information.

I understand that if I have any questions or concerns regarding my child's participation in this research project, I may contact Sarah Woodruff (455-4348) or Dr. Connie BothwellMyers (453-5035 or cbm@unb.ca).

I understand should I decide that my child could benefit from a movement education program, the researchers are able to facilitate a program through the Faculty of Kinesiology at the University of New Brunswick.

I understand that my child may withdraw from this study at any time.

I have read and understood all the above conditions. I give consent for my child to participate in this research project.

Please print your child's name:

Please print your own name:

Your signature:

Date:

Your child's school:

Please check Yes, if you are interested in receiving a summary of the results of this research study.

Yes, I am interested in receiving a summary of the results of this research study.

No, I am not interested in receiving a summary of the results of this research study.

Name:

Address:

Phone:

(h)

(w)

Email: 
Appendix F: Children who were excluded from the Training Set 
Table F.1

Children who were excluded from the training set

\begin{tabular}{|c|c|}
\hline 8001 - outlier values & 8130 - Deleted from Wilson (1998) \\
\hline 8003 - missing data & 8141 - missing data \\
\hline 8006 - Deleted from Wilson (1998) & 8145 - outlier values \\
\hline 8007 - outlier values & 8152 - outlict values \\
\hline 8008 - outlier values & 8154 - Wilson (1998) deleted right side \\
\hline 8009 - outlier values & 8155 - outlier values \\
\hline 8010 - outlier values & 8158 - missing data \\
\hline 8014 - outlier values & $8160-$ outlier values \\
\hline 8016 - outlier values & 8162 - outlier values \\
\hline 8030 - Wilson (1998) deleted left side & 8164 - missing data \\
\hline 8035 - outlier values & 8171 - outlier values \\
\hline 8040 - outlier values & 8174 - Wilson (1998) deleted right side \\
\hline 8044 - missing data & 8185 - outlier values \\
\hline 8045 - outlier values & 8190 - missing data \\
\hline 8051 - outlier values & 8205 - missing data \\
\hline 8054 - outlier values & 8222 - outlier values \\
\hline 8061 - missing data & 8229 - outlier values \\
\hline 8066 - outlier values & 8230 - missing data \\
\hline 8068 - outlier values & 8231 - Wilson (1998) deleted left side \\
\hline 8069 - missing data & 8237 - outlier values \\
\hline 8071 - outlier values & 8248 - outlier values \\
\hline 8101 - outlier values & 8287 - missing data \\
\hline 8103 - missing data & 8295 - outlier values \\
\hline 8112 - outlier values & 8313 - missing data \\
\hline 8125 - outlier values & \\
\hline
\end{tabular}


Appendix G: Correlation Matrices 
Table G.1

Correlation Matrix for Left Side

\begin{tabular}{ccccc}
\hline Variables & Opposite Toe & Single & Toe Off & Step \\
& Off $(\%)$ & Stance (\%) & $(\%)$ & Length (\%) \\
\hline Opposite Toe Off (\%) & 1 & & & \\
Single Stance (\%) & -0.843 & 1 & & \\
Toe Off $(\%)$ & 0.759 & -0.603 & 1 & \\
Step Length (\%) & -0.067 & 0.087 & 0.009 & 1 \\
\hline Note. $\mathrm{n}=139$ & & & &
\end{tabular}

Table G.2

Correlation Matrix for Right Side

\begin{tabular}{ccccc}
\hline Variables & $\begin{array}{c}\text { Opposite Toe } \\
\text { Off }(\%)\end{array}$ & Single & Toe Off & Step \\
& 1 & & & \\
\hline Opposite Toe Off $(\%)$ & -0.884 & 1 & & \\
Single Stance (\%) & 0.77 & -0.629 & 1 & \\
Toe Off $(\%)$ & 0.057 & -0.074 & 0.099 & 1 \\
\hline Step Length $(\%)$ & & & & \\
\hline
\end{tabular}

Note. $n=139$ 
Table G.3

Correlation Matrix for Combined Left and Right Sides

\begin{tabular}{ccccc}
\hline Variables & $\begin{array}{c}\text { Opposite Toe } \\
\text { Off (\%) }\end{array}$ & $\begin{array}{c}\text { Single } \\
\text { Stance (\%) }\end{array}$ & $\begin{array}{c}\text { Toe Off } \\
(\%)\end{array}$ & $\begin{array}{c}\text { Step } \\
\text { Length (\%) }\end{array}$ \\
\hline Opposite Toe Off (\%) & 1 & & & \\
Single Stance (\%) & -0.861 & 1 & & \\
Toe Off (\%) & 0.762 & -0.613 & 1 & \\
Step Length (\%) & 0.011 & 0.016 & 0.046 & 1 \\
\hline
\end{tabular}

Note. $n=278$ 
Appendix H: Inverse Covariance Matrix 
Table H.l

Inverse Covariance Matrix for both Left and Right Sides

\begin{tabular}{ccccc}
\hline Variables & $\begin{array}{c}\text { Opposite Toe } \\
\text { Off (\%) }\end{array}$ & $\begin{array}{c}\text { Single } \\
\text { Stance (\%) }\end{array}$ & $\begin{array}{c}\text { Toe Off } \\
(\%)\end{array}$ & $\begin{array}{c}\text { Step } \\
\text { Length (\%) }\end{array}$ \\
\hline Opposite Toe Off (\%) & 2.16 & 1.38 & -0.8 & -0.01 \\
Single Stance (\%) & 1.38 & 1.48 & -0.15 & -0.04 \\
Toe Off (\%) & -0.8 & -0.15 & 0.87 & -0.04 \\
Step Length (\%) & -0.01 & -0.04 & -0.04 & 0.66 \\
\hline
\end{tabular}

Note. $n=278$ 
Appendix I: Missing Data 
Table I.1

Number of Missing Data Points for the Trials that were Processed using the Wilson Score

\begin{tabular}{lccccc}
\hline ID & Side & Total Number & Hip & Knee & Ankle \\
\hline DCD4 & L & 55 & 0 & 4 & 11 \\
DCD5 & R & 45 & 0 & 0 & 1 \\
DCD6 & L & 47 & 0 & 0 & 0 \\
DCD6 & R & 49 & 0 & 11 & 11 \\
DCD7 & L & 51 & 0 & 0 & 0 \\
DCD7 & R & 48 & 0 & 5 & 13 \\
\hline
\end{tabular}

Table 1.2

Number of Missing Data Points for the Trials that were not Processed for the Wilson $\underline{\text { Score }}$

\begin{tabular}{lccccc}
\hline ID & Side & Total Number & Hip & Knee & Ankle \\
\hline DCD1 & L & 53 & 29 & 37 & 39 \\
DCD1 & R & 47 & 0 & 24 & 26 \\
DCD2 & L & 58 & 39 & 46 & 21 \\
DCD2 & R & 59 & 19 & 22 & 59 \\
DCD3 & L & 50 & 20 & 47 & 34 \\
DCD3 & R & 46 & 32 & 39 & 38 \\
DCD4 & R & 62 & 38 & 62 & 46 \\
DCD5 & L & 49 & n/a & n/a & n/a \\
\hline
\end{tabular}

Note. $\mathrm{n} / \mathrm{a}$ refers to a suspected recording error 
Appendix J: Standardized $\underline{B}^{(i)}$ Vectors 


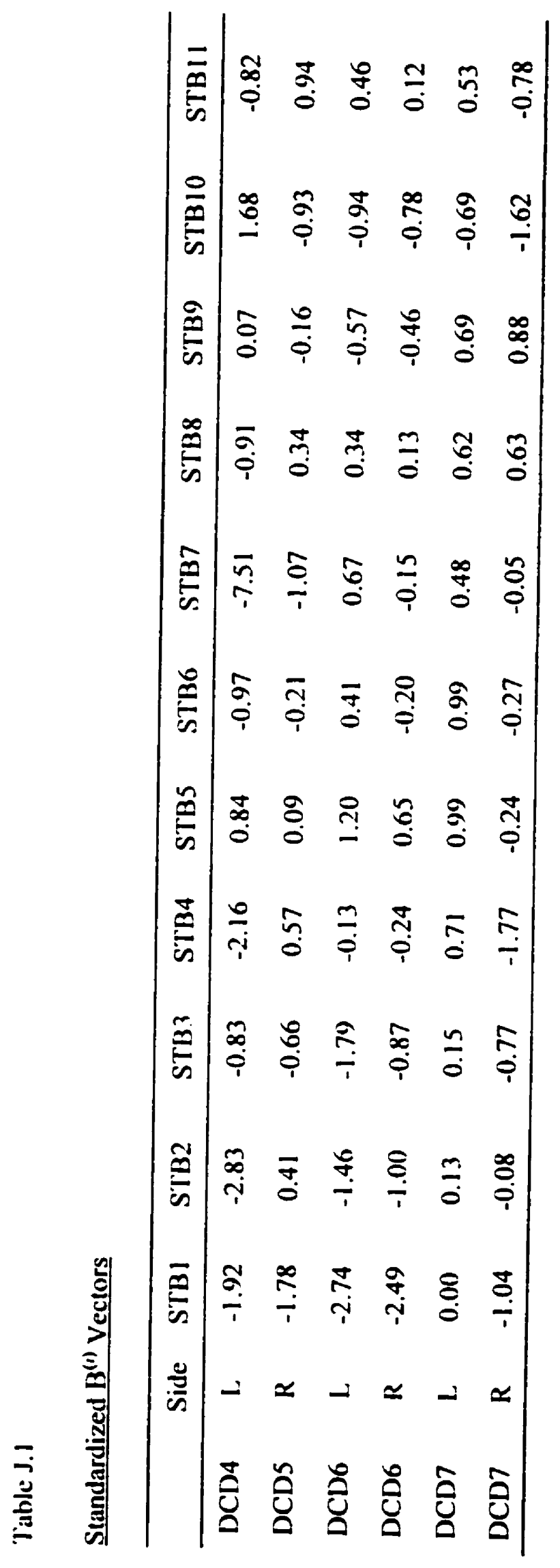


Appendix K: Covariance Matrices for the San Diego Database

Study and Children with DCD 
Table K.1

Covariance Structure of San Diego Children's Hospital Normative Time/Distance Measurements (Correlation Matrix in Brackets)

\begin{tabular}{ccccc}
\hline Variables & $\begin{array}{c}\text { Opposite Toe } \\
\text { Off (\%) }\end{array}$ & $\begin{array}{c}\text { Single } \\
\text { Stance (\%) }\end{array}$ & $\begin{array}{c}\text { Toe Off } \\
(\%)\end{array}$ & $\begin{array}{c}\text { Step } \\
\text { Length (\%) }\end{array}$ \\
\hline Opposite Toe Off (\%) & 2.71 & -2.3 & 2.1 & 0.02 \\
Single Stance (\%) & -2.30 & 2.65 & -1.67 & 0.03 \\
& $(-0.861)$ & & & \\
Toe Off (\%) & 2.10 & -1.67 & 2.81 & 0.1 \\
& $(0.762)$ & $(-0.613)$ & & \\
Step Length (\%) & 0.02 & 0.03 & 0.10 & 1.53 \\
& $(0.011)$ & $(0.016)$ & $(0.046)$ & \\
\hline
\end{tabular}

Note. $n=278$ 
Table K.2

Covariance Structure of Children with DCD Time/distance Measurements (Correlation Matrix in Brackets)

\begin{tabular}{ccccc}
\hline Variables & $\begin{array}{c}\text { Opposite Toe } \\
\text { Off (\%) }\end{array}$ & $\begin{array}{c}\text { Single } \\
\text { Stance (\%) }\end{array}$ & $\begin{array}{c}\text { Toe Off } \\
(\%)\end{array}$ & $\begin{array}{c}\text { Step } \\
\text { Length (\%) }\end{array}$ \\
\hline Opposite Toe Off (\%) & 18.9 & -8.55 & 13.85 & -16.7 \\
Single Stance (\%) & -8.55 & 9.87 & -3.67 & 9.92 \\
& $(-0.626)$ & & & \\
Toe Off (\%) & 13.85 & -3.67 & 14.64 & -17.05 \\
& $(0.833)$ & $(-0.306)$ & & \\
Step Length (\%) & -16.70 & 9.92 & -17.05 & 42.86 \\
& $(-0.587)$ & $(0.483)$ & $(-0.681)$ & \\
\hline
\end{tabular}

Note. $\mathrm{n}=11$ 